\title{
COMPUTING THE INVARIANT MEASURE AND THE LYAPUNOV EXPONENT FOR ONE-DIMENSIONAL MAPS USING A MEASURE-PRESERVING POLYNOMIAL BASIS
}

\author{
PHILIP J. ASTON AND OLIVER JUNGE
}

\begin{abstract}
We consider a generalisation of Ulam's method for approximating invariant densities of one-dimensional maps. Rather than use piecewise constant polynomials to approximate the density, we use polynomials of degree $n$ which are defined by the requirement that they preserve the measure on $n+1$ neighbouring subintervals. Over the whole interval, this results in a discontinuous piecewise polynomial approximation to the density. We prove error results where this approach is used to approximate smooth densities. We also consider the computation of the Lyapunov exponent using the polynomial density and show that the order of convergence is one order better than for the density itself. Together with using cubic polynomials in the density approximation, this yields a very efficient method for computing highly accurate estimates of the Lyapunov exponent. We illustrate the theoretical findings with some examples.
\end{abstract}

\section{INTRODUCTION}

For dynamical systems with complicated ("chaotic") dynamical behavior, a computational investigation based on simulation is often not appropriate. This is caused by the fact that due to the sensitive dependence of individual trajectories on their initial condition a long-term simulation is ill-conditioned and quickly amplifies round-off errors in a way that renders the computed values meaningless. In fact, for these systems, a computational approach which explicitly accounts for uncertainties is more appropriate. Thus, instead of considering the evolution of a single state, one investigates the evolution of a given density on state space. This evolution is given by the Frobenius-Perron (or more generally the transfer) operator of the system, a linear operator on a suitably chosen function space (e.g., some $L^{p}$ or BV space). In fact, it turns out [13] that in a suitable setting, the spectrum and the associated eigenfunctions of this operator allow a complete statistical description of the dynamics. This has been used extensively in order to design a new computational approach to the identification of conformations of (bio-)molecules, as first proposed in [15] and subsequently worked out in, e.g., [16, 51] (see also the references therein). Another important emerging field of application is in the description of transport phenomena in, for example, dynamical astronomy [14] or fluid flows 31,32 .

In particular, a fixed point of this operator yields a ("natural") invariant measure (resp. density) of the system. Roughly speaking, a natural invariant measure $\mu$ characterizes the long term dynamics in the sense that for a given subset $A$ of

Received by the editor November 23, 2011 and, in revised form, September 17, 2012.

2010 Mathematics Subject Classification. Primary 37M25, 65P20. 
state space, $\mu(A)$ is the average number of times that one observes the system in $A$ (as stated by the Birkhoff Ergodic Theorem). This property allows for various applications, for example in the design of drilling bits [44, but also in number theory, such as the Kronecker-Weyl or Borel's normal number theorem [3,42. Furthermore, there is an intrinsic connection between the (if unique) natural invariant measure and various other dynamical system quantities like Lyapunov exponents, entropy or dimensions [2,9].

In most cases, however, even for simple one-dimensional systems, there will be no analytic description of $\mu$ (resp. its density) available and it is therefore of interest to obtain numerical approximations. A common approach is to discretize the transfer operator and to compute fixed points of the resulting matrix. As mentioned earlier, other eigenvalues and eigenfunctions might also be of interest. In this paper, we propose a new approach to this discretization which is based on a measure preserving polynomial basis.

To be more precise, suppose that a one-dimensional piecewise smooth map $g$ : $I \rightarrow I$ on a compact interval $I \subset \mathbb{R}$ has a unique chaotic attractor with an absolutely continuous invariant measure $\mu$. We consider piecewise polynomial approximations to the density $d$ associated with $\mu$. To this end, we define a partition of the interval $I$ by dividing it into a subintervals $I_{i}=\left[x_{i-1}, x_{i}\right], i=1,2, \ldots, N$ of equal length $h=\left(x_{N}-x_{0}\right) / N$. For each subinterval, we define

$$
m_{i}=\int_{I_{i}} d \mu, \quad i=1,2, \ldots, N .
$$

Thus, $m_{i}$ is the mass of the interval $I_{i}$ with respect to the invariant measure $\mu$. Clearly we have the property that

$$
\sum_{i=1}^{N} m_{i}=1,
$$

since $\mu$ is a probability measure. We want to approximate the density $d$ of $\mu$ given that the only information that we have about $\mu$ is the masses $m_{i}, i=1,2, \ldots, N$.

For approximating invariant densities, Ulam's classical method [53] consists in using piecewise constant approximations on each subinterval with the constants being found by computing a fixed point (i.e., an eigenvector at the eigenvalue 1) of a (discretized) Markov operator, the so-called Frobenius-Perron operator or more generally transfer operator. This operator describes how measures (and densities) are pushed forward under the dynamics of the map $g$. Ulam's method has been proved to be convergent for piecewise expanding maps [45]. Since then much effort has gone into analysing the method [4, 10, 18, 20, 21, 26], computational questions [19, 36, extending the proof to more general classes of transformations [22, 28, 30, 37, 39, 47] or extending the method to higher dimensional systems [11, $13,24,27,40$, 41.

For nonsmooth invariant densities, a piecewise constant approximation may be a good choice. However, in the smooth case (for example, if the map is perturbed by white noise), it would be better to use a higher order approximation. Higher order polynomial approximations to the invariant density have been obtained by Ding and coworkers [17, 21, 23, 25. They used a Galerkin method for finding a piecewise polynomial approximation to the density but did not go on to consider the computation of the Lyapunov exponent. 
Increasingly, numerical methods are being developed which incorporate and preserve features of the problem being considered. One well-known example of this is geometric integrators for ODEs which encode properties of the ODEs, such as a symplectic structure or conservation of an invariant, into the numerical method 34 . Our approach to the problem of computing an invariant density is based on this philosophy. The essence of the density is that the measure should be preserved on any subinterval and so we construct a discontinuous piecewise polynomial basis which is defined locally by the requirement that the measure is preserved on neighbouring intervals. Combining many such polynomials over the whole interval, the coefficients of the approximate invariant density with respect to this basis can again be found by computing a fixed point of the associated discrete Frobenius-Perron operator. Using this new basis, which turns out to lead to a Petrov-Galerkin discretisation of the Frobenius-Perron operator, we improve upon the convergence rates in [17, 21] for piecewise quadratic approximations. In addition, we consider the computation of the Lyapunov exponent using the invariant density and give corresponding error estimates. It turns out (see Theorem 7.1) that using our measure preserving basis, one actually gains one order in the convergence of the Lyapunov exponent. Using cubic polynomials, this leads to a highly efficient method for computing estimates of the Lyapunov exponent.

An outline of the paper follows: In Section 2, we derive the measure-preserving polynomial basis and perform an error analysis for a density that is approximated by such a polynomial. In Section 3, we describe how this piecewise polynomial approximation to the density can be found by solving the Frobenius-Perron equation. Section 4 considers the problem of integration when the density is replaced by a polynomial while Section 5 gives an error analysis when the density is found as a solution of the discretised Frobenius-Perron fixed point equation. Some examples are used to illustrate the theory in Section 6. The computation of the Lyapunov exponent using the polynomial approximation to the density is considered in Section 7, and the errors in the Lyapunov exponent are derived. These results are illustrated for the examples considered earlier. Finally, in Section 8, two extensions of this work are described.

\section{Measure-Preserving polynomial approximations to the Density}

Our aim is to construct a (discontinuous) piecewise polynomial approximation to an invariant density. To do this, we start by considering the problem of obtaining a polynomial approximation to the density function over a subset of $n+1$ adjacent intervals $\left[x_{i}, x_{i+n+1}\right]=\bigcup_{j=0}^{n} I_{i+j+1}$ for some $i$. As with Gaussian quadrature, we prefer to work with the standard interval $[-1,1]$ and so we perform a change of variables to map the interval $\left[x_{i}, x_{i+n+1}\right]$ onto $[-1,1]$ using

$$
t=t_{n, i}(x)=-1+\frac{2\left(x-x_{i}\right)}{(n+1) h} .
$$

The corresponding inverse transformation is given by

$$
x=x_{n, i}(t)=\frac{1}{2}(n+1) h(t+1)+x_{i} .
$$

We transform the points $x_{i+j}$ onto the interval $[-1,1]$ by

$$
t_{j}=t_{n, i}\left(x_{i+j}\right)=-1+\frac{2 j}{n+1}, \quad j=0,1, \ldots, n+1,
$$


assuming that the points are evenly spaced. We also define a new density function $D_{n, i}(t)$ which satisfies

$$
D_{n, i}(t) d t=d(x) d x, \quad x \in\left[x_{i}, x_{i+n+1}\right]
$$

which implies that

$$
D_{n, i}(t)=\frac{d x}{d t} d\left(x_{n, i}(t)\right)=\frac{1}{2}(n+1) h d\left(x_{n, i}(t)\right),
$$

using (2.2). Using this definition, we find that

$$
\int_{x_{i}}^{x_{i+n+1}} f(x) d(x) d x=\int_{-1}^{1} F_{n, i}(t) D_{n, i}(t) d t,
$$

where $F_{n, i}(t)=f\left(x_{n, i}(t)\right)$. Defining

$$
M_{j}=m_{i+j+1}, \quad j=0, \ldots, n,
$$

we obtain

$$
M_{j}=m_{i+j+1}=\int_{x_{i+j}}^{x_{i+j+1}} d(x) d x=\int_{t_{j}}^{t_{j+1}} D_{n, i}(t) d t, \quad j=0, \ldots, n .
$$

We now use the $n+1$ values $M_{j}, j=0, \ldots, n$ to obtain a polynomial $p_{n, i}(t) \in \Pi_{n}$, where $\Pi_{n}$ is the space of polynomials with real coefficients of degree at most $n$, which approximates the density function $D_{n, i}(t)$ which can be used in the integration formula (2.5) with a higher order Gaussian quadrature method to obtain higher accuracy for the integral. The criterion which we use to define the polynomial is that it should preserve the measure on each subinterval, that is,

$$
\int_{t_{j}}^{t_{j+1}} p_{n, i}(t) d t=M_{j}, \quad j=0,1, \ldots, n .
$$

We will consider two different methods for determining the measure-preserving polynomial $p_{n, i}(t)$, but we first establish uniqueness.

Lemma 2.1. The polynomial $p_{n, i}(t) \in \Pi_{n}$ satisfying (2.7) is unique.

Proof. Assume that $p_{n, i}(t)$ and $q_{n, i}(t)$ are both polynomials that satisfy the conditions (2.7) and define

$$
h_{n, i}(t)=p_{n, i}(t)-q_{n, i}(t) .
$$

Then $h_{n, i}(t) \in \Pi_{n}$ and satisfies the conditions

$$
\int_{t_{j}}^{t_{j+1}} h_{n, i}(t) d t=0, \quad j=0,1, \ldots, n .
$$

These conditions imply that, for each value of $j$, there is at least one point $\tau_{j} \in$ $\left(t_{j}, t_{j+1}\right)$ such that $h_{n, i}\left(\tau_{j}\right)=0$. Thus, in total, there are at least $n+1$ distinct points in the interval $(-1,1)$ at which $h_{n}$ vanishes. The only polynomial of degree $n$ that has at least $n+1$ distinct zeros is the zero polynomial, and so $h_{n, i}(t)=0$, giving $p_{n, i}(t)=q_{n, i}(t)$.

There are two approaches which can be used to determine the polynomial $p_{n, i}(t)$, both of which will be useful later. 
2.1. Constructing the measure-preserving polynomial by interpolating the measure. We define the measure $\nu_{n, i}$ associated with the density function $D_{n, i}(t)$ by

$$
\nu_{n, i}(t)=\int_{-1}^{t} D_{n, i}(\tau) d \tau
$$

and we note that

$$
\nu_{n, i}\left(t_{j}\right)=\sum_{k=0}^{j-1} M_{k}, \quad j=1,2, \ldots, n+1, \quad \nu_{n, i}\left(t_{0}\right)=0 .
$$

Strictly speaking, $\nu_{n, i}$ is not a probability measure since $\int_{-1}^{1} d \nu_{n, i} \neq 1$, but it is a local piece of the probability measure $\mu$. From (2.9), we have $n+2$ values of the function $\nu_{n, i}(t)$ which we can interpolate using a polynomial $q_{n+1, i}(t) \in \Pi_{n+1}$. The derivative of this polynomial will give an approximation to the density function which satisfies (2.7). Thus, we define

$$
p_{n, i}(t)=q_{n+1, i}^{\prime}(t),
$$

where prime denotes differentiation with respect to $t$. Using the Lagrange form of the interpolating polynomial [7, we thus have that

$$
\begin{aligned}
q_{n+1, i}(t) & =\sum_{j=1}^{n+1} \nu_{n, i}\left(t_{j}\right) L_{n, j}(t) \\
& =\sum_{j=1}^{n+1} \sum_{k=0}^{j-1} M_{k} L_{n, j}(t) \\
& =\sum_{k=0}^{n} M_{k} \sum_{j=k+1}^{n+1} L_{n, j}(t)
\end{aligned}
$$

where

$$
L_{n, j}(t)=\prod_{\substack{i=0 \\ i \neq j}}^{n}\left(\frac{t-t_{i}}{t_{j}-t_{i}}\right) .
$$

Note that there is no term in the definition of $q_{n+1, i}(t)$ with $j=0$ since $\nu_{n, i}\left(t_{0}\right)=0$. Thus, one representation of the polynomial which satisfies the conditions (2.7) is

$$
p_{n, i}(t)=\sum_{k=0}^{n} M_{k} \sum_{j=k+1}^{n+1} L_{n, j}^{\prime}(t)
$$

2.2. Constructing the measure-preserving polynomial using an appropriate basis. The derivatives of the basis functions $L_{n, j}(t)$ in the previous method are clearly quite messy to evaluate. An alternative approach is to seek the polynomial $p_{n, i}(t)$ in the form

$$
p_{n, i}(t)=\sum_{k=0}^{n} M_{k} \ell_{n, k}(t) .
$$

The basis functions $\ell_{n, k}(t)$ can be found by substituting this form of the polynomial into the conditions (2.7) and equating coefficients of $M_{k}$. This implies that for each 
TABLE 1. Basis functions for the measure-preserving polynomial approximation to the density.

\begin{tabular}{|l|l|}
\hline$n=0$ & $\ell_{0,0}(t)=\frac{1}{2}$ \\
\hline$n=1$ & $\ell_{1,0}(t)=-t+\frac{1}{2}$ \\
& $\ell_{1,1}(t)=\ell_{1,0}(-t)$ \\
\hline$n=2$ & $\ell_{2,0}(t)=\frac{1}{16}\left(27 t^{2}-18 t-1\right)$ \\
& $\ell_{2,1}(t)=\frac{1}{8}\left(-27 t^{2}+13\right)$ \\
& $\ell_{2,2}(t)=\ell_{2,0}(-t)$ \\
\hline$n=3$ & $\ell_{3,0}(t)=\frac{1}{6}\left(-16 t^{3}+12 t^{2}+2 t-1\right)$ \\
& $\ell_{3,1}(t)=\frac{1}{6}\left(48 t^{3}-12 t^{2}-30 t+7\right)$ \\
& $\ell_{3,2}(t)=\ell_{3,1}(-t)$ \\
& $\ell_{3,3}(t)=\ell_{3,0}(-t)$ \\
\hline
\end{tabular}

$k=0,1, \ldots, n$, we have the $n+1$ conditions

$$
\int_{t_{j}}^{t_{j+1}} \ell_{n, k}(t) d t=\left\{\begin{array}{cc}
0, & k \neq j, \\
1, & k=j,
\end{array} \quad j=0,1, \ldots, n .\right.
$$

These equations represent a system of linear equations for the $n+1$ coefficients in the polynomial $\ell_{n, k}(t)$.

Of course this is only a different representation of the same polynomial found using the measure and so

$$
\ell_{n, k}(t)=\sum_{j=k+1}^{n+1} L_{n, j}^{\prime}(t) .
$$

The basis functions defined by the conditions (2.11) for some low values of $n$ are listed in Table1. The three basis functions for the case $n=2$ are shown in Figure 1. Some properties of these basis functions can be determined.

Lemma 2.2. The basis functions $\ell_{n, k}(t)$ have the following properties:

(i) $\ell_{n, n-k}(t)=\ell_{n, k}(-t)$;

(ii) $\int_{-1}^{1} \ell_{n, k}(t) d t=1$;

(iii) $\sum_{k=0}^{n} \ell_{n, k}(t)=\frac{n+1}{2}$;

(iv) The function $\ell_{n, k}(t)$ has $n$ simple roots with one root in each of the intervals $\left(t_{j}, t_{j+1}\right)$ for $j \neq k$.

Proof. Results (i) and (ii) follow directly from the definition of the basis functions. For the third result, consider the case that $D_{n, i}(t)=c$ for some constant $c$. Then $M_{i}=2 c /(n+1), i=0, \ldots, n$ and $p_{n, i}(t)=D_{n, i}(t)=c$. Substituting these into (2.10) gives the required result. For the fourth result, note that the condition that the integral of $\ell_{n, k}(t)$ is zero over the subinterval $\left[t_{j}, t_{j+1}\right]$ implies that there must 


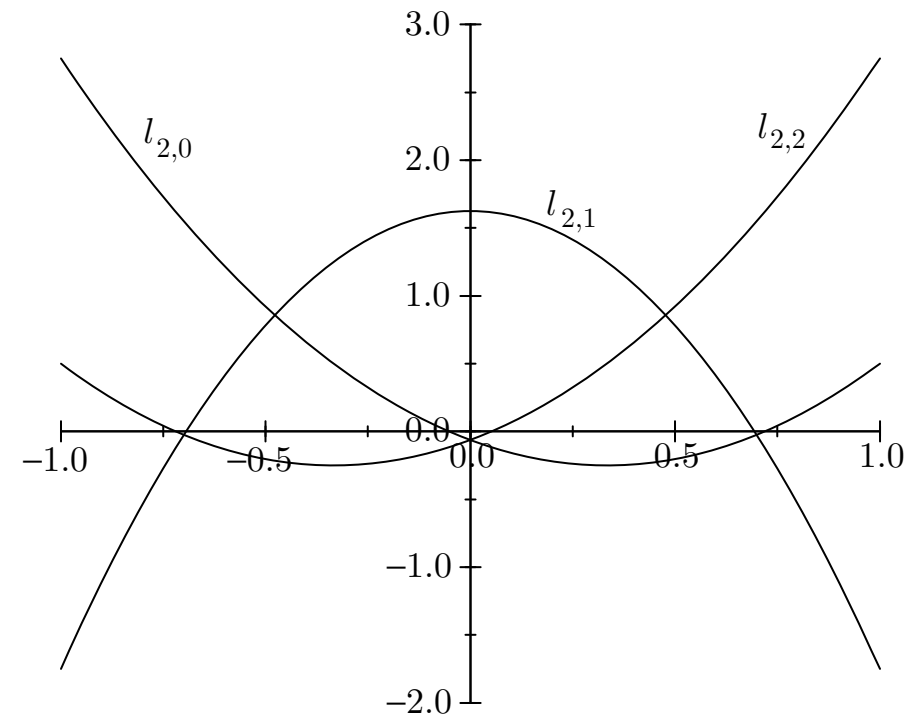

FiguRE 1. The three basis functions for $n=2$.

be at least one point in $\left(t_{j}, t_{j+1}\right)$ at which the function is zero by the first Mean Value Theorem for Integrals. Since one root in each interval gives us the maximum possible number of roots, then there must be precisely one root in each interval.

2.3. Error analysis. Having determined methods for finding a polynomial approximation $p_{n, i}(t)$ to the density function $D_{n, i}(t)$, we must now consider the errors in this approximation. Thus, we write

$$
D_{n, i}(t)=p_{n, i}(t)+e_{n, i}(t),
$$

for some error function $e_{n, i}(t)$.

Using the first approach of interpolating the measure, the error in the interpolating polynomial $q_{n+1, i}(t)$ is given by

$$
\nu_{n, i}(t)=q_{n+1, i}(t)+\varepsilon_{n+1, i}(t),
$$

where

$$
\varepsilon_{n+1, i}(t)=\frac{\nu_{n, i}^{(n+2)}\left(\kappa_{n, i}(t)\right)}{(n+2) !} \prod_{j=0}^{n+1}\left(t-t_{j}\right),
$$

for some $\kappa_{n, i}(t) \in(-1,1)$, assuming that $\nu_{n, i}(t) \in C^{n+2}[-1,1]$ (see [7]). However, to obtain the error in the density requires differentiating $\varepsilon_{n+1, i}(t)$ which does not lead to a nice form for $e_{n, i}(t)$. Thus, we also derive the error based on the alternative approach of determining the polynomial in terms of the basis functions $\ell_{n, k}(t)$.

Integrating (2.12) over one subinterval and using (2.7) gives that

$$
\int_{t_{j}}^{t_{j+1}} e_{n, i}(t) d t=0, \quad j=0,1, \ldots, n .
$$

By the first Mean Value Theorem for Integrals, this implies that there is at least one point $\tau_{j} \in\left(t_{j}, t_{j+1}\right)$ such that $e_{n, i}\left(\tau_{j}\right)=0, j=0,1, \ldots, n$ and so there are at 
least $n+1$ distinct points in the interval $(-1,1)$ at which the polynomial interpolates the density. If we choose precisely one such point in each subinterval, then this interpolation problem defines a unique polynomial in $\Pi_{n}$ which must be the polynomial $p_{n, i}(t)$. Thus, regarding the problem as an interpolation problem, we can express the error function as

$$
e_{n, i}(t)=\frac{D_{n, i}^{(n+1)}\left(\xi_{n, i}(t)\right)}{(n+1) !} \prod_{j=0}^{n}\left(t-\tau_{j}\right),
$$

where $\xi_{n, i}(t) \in(-1,1)$ assuming that $D_{n, i}(t) \in C^{n+1}[-1,1]$ (see [7]). Note that the points $\tau_{j}$ depend on the particular function $D_{n, i}(t)$.

Since there is a simple relationship (2.8) between $\nu_{n, i}(t)$ and $D_{n, i}(t)$, there must also be a similar relationship between the errors, which is given by

$$
\varepsilon_{n+1, i}(t)=\int_{-1}^{t} e_{n, i}(\tau) d \tau
$$

\section{Computing the polynomial Density From the Frobenius-PERRON EQUATION}

Using the approach described in the previous section, we have reduced the description of the invariant density associated with an iteration function $g: I \rightarrow I$ to a finite dimensional approximation which is characterised by the $N$-dimensional vector $\mathbf{m}$ whose components are

$$
m_{i}=\int_{I_{i}} d \mu, \quad i=1,2, \ldots, N .
$$

Thus, given a vector $\mathbf{m}$, a discontinuous piecewise polynomial approximation to the density can be constructed, which is given by

$$
d_{N}^{n}(x)=\sum_{i=1}^{N} m_{i} b_{i}(x)
$$

where $b_{i}(x), i=1, \ldots, N$ are the piecewise polynomial basis functions which were derived in the previous section, mapped back onto the appropriate subintervals. However, the problem is that we do not know the vector $\mathbf{m}$. Thus, we use this polynomial approximation to the density and substitute it into the Frobenius-Perron equation, which gives an eigenvalue problem from which the vector $\mathbf{m}$ can be found.

The Frobenius-Perron equation is given by

$$
\int_{J} d \mu=\int_{g^{-1}(J)} d \mu
$$

for any interval $J \subset I$. To obtain a set of determining equations for the vector m, we use the Frobenius-Perron equation with $J=I_{i}, i=1,2, \ldots, N$ giving the equations

$$
\int_{I_{i}} d \mu=\int_{g^{-1}\left(I_{i}\right)} d \mu, \quad i=1,2, \ldots, N
$$

In terms of the invariant density, these equations are given by

$$
\int_{I_{i}} d(x) d x=\int_{g^{-1}\left(I_{i}\right)} d(x) d x, \quad i=1,2, \ldots, N .
$$


Approximating the invariant density $d$ by the piecewise polynomial function $d_{N}^{n}$, the equations become

$$
\int_{I_{i}} d_{N}^{n}(x) d x=\int_{g^{-1}\left(I_{i}\right)} d_{N}^{n}(x) d x, \quad i=1,2, \ldots, N
$$

Now, by construction,

$$
\int_{I_{i}} d_{N}^{n}(x) d x=m_{i}, \quad i=1, \ldots, N .
$$

Similarly, the right-hand side of (3.2) can be written as

$$
\begin{aligned}
\int_{g^{-1}\left(I_{i}\right)} d_{N}^{n}(x) d x & =\bigcup_{k=1}^{K} \int_{x_{L, k}}^{x_{R, k}} d_{N}^{n}(x) d x \\
& =\bigcup_{k=1}^{K} \int_{x_{L, k}}^{x_{R, k}} \sum_{j=1}^{N} m_{j} b_{j}(x) d x \\
& =\sum_{j=1}^{N} m_{j} \bigcup_{k=1}^{K} \int_{x_{L, k}}^{x_{R, k}} b_{j}(x) d x
\end{aligned}
$$

where $K$ is the number of preimages of the interval $I_{i}$, and for each $k=1, \ldots, K$, $g\left(x_{L, k}\right)=x_{i-1}, g\left(x_{R, k}\right)=x_{i}$. Thus, the equations (3.2) can be written as the linear system

$$
\mathbf{m}=P_{N}^{n} \mathbf{m}
$$

where

$$
P_{N i, j}^{n}=\bigcup_{k=1}^{K} \int_{x_{L, k}}^{x_{R, k}} b_{j}(x) d x .
$$

Thus, the vector $\mathbf{m}$ is found as the eigenvector of the matrix $P_{N}^{n}$ associated with the eigenvalue 1 , together with the normalisation condition (1.2).

We note that when $n=0$ (piecewise constant polynomial approximations), this is the standard Ulam method for approximating the invariant density [53].

\section{Integration}

There are two different types of integral that we need when solving for the invariant density and when computing the Lyapunov exponent and so we consider them both at this stage. For both integrals, we replace the density by the piecewise polynomial approximation and provide an error analysis associated with this approximation.

4.1. Case I. When computing the density using the Frobenius-Perron equation, as described in Section 3, integrals of the form

$$
\int_{g^{-1}\left(I_{i}\right)} d(x) d x
$$

must be evaluated (see (3.1)), where we recall that there may be several preimages of the interval $I_{i}$. Each preimage of $I_{i}$ may be contained in a single interval $I_{k}$ for 
some $k$, or it may contain some whole intervals and only parts of others. Thus, we can write

$$
\int_{g^{-1}\left(I_{i}\right)} d(x) d x=\sum_{j=0}^{J-1} \int_{g^{-1}\left(I_{i}\right) \cap I_{k+j}} d(x) d x
$$

for some $k$, assuming that $g^{-1}\left(I_{i}\right) \cap I_{k+j} \neq \emptyset$ for $j=0, \ldots, J-1$. If $J \geq 2$, then we obtain

$\sum_{j=0}^{J-1} \int_{g^{-1}\left(I_{i}\right) \cap I_{k+j}} d(x) d x=\int_{g^{-1}\left(I_{i}\right) \cap I_{k}} d(x) d x+\sum_{j=1}^{J-2} m_{k+j}+\int_{g^{-1}\left(I_{i}\right) \cap I_{k+J-1}} d(x) d x$

whereas, if $J=1$, then

$$
\int_{g^{-1}\left(I_{i}\right)} d(x) d x=\int_{g^{-1}\left(I_{i}\right) \cap I_{k}} d(x) d x .
$$

Thus, we have to compute integrals of the form

$$
\int_{x_{\ell}}^{x_{r}} d(x) d x
$$

where $x_{\ell}$ or $x_{r}$ may be one of the mesh points, but not both (when $J \geq 2$ ), or neither of the points is a mesh point (when $J=1$ ). Using our change of variables to map onto the standard interval $[-1,1]$, we then have that

$$
\int_{x_{\ell}}^{x_{r}} d(x) d x=\int_{t_{\ell}}^{t_{r}} D_{n, i}(t) d t
$$

where $t_{\ell}=t_{n, i}\left(x_{\ell}\right), t_{r}=t_{n, i}\left(x_{r}\right)$ and $\left[t_{\ell}, t_{r}\right] \subseteq[-1,1]$, for some $i$.

We assume in this section that the measures $m_{i}$ on the intervals $I_{i}, i=1, \ldots, N$ are known exactly and we consider only the errors associated with the piecewise polynomial approximation to the measure. Using the polynomial approximation to the density then gives the following result.

Theorem 4.1. If $D_{n, i}(t) \in C^{n+1}[-1,1]$ and (1.1) is satisfied, then

$$
\begin{gathered}
\int_{t_{\ell}}^{t_{r}} D_{n, i}(t) d t=\int_{t_{\ell}}^{t_{r}} p_{n, i}(t) d t+O\left(h^{n+2}\right), \\
\int_{t_{\ell}}^{t_{r}}\left|D_{n, i}(t)-p_{n, i}(t)\right| d t=O\left(h^{n+2}\right) .
\end{gathered}
$$

Proof. Integrating the polynomial approximation with error given by (2.12), we obtain

$$
\int_{t_{\ell}}^{t_{r}} D_{n, i}(t) d t=\int_{t_{\ell}}^{t_{r}} p_{n, i}(t) d t+\int_{t_{\ell}}^{t_{r}} e_{n, i}(t) d t
$$

Thus, for (4.1), we need to estimate

$$
\int_{t_{\ell}}^{t_{r}} e_{n, i}(t) d t
$$

Similarly, for (4.2), we need to estimate

$$
\int_{t_{\ell}}^{t_{r}}\left|e_{n, i}(t)\right| d t .
$$


From (2.15), we see that the function $e_{n, i}(t)$ involves $D_{n, i}^{(n+1)}\left(\xi_{n, i}(t)\right)$. Now from (2.4), we know that

$$
D_{n, i}(t)=\operatorname{chd}\left(x_{n, i}(t)\right)
$$

where $c=\frac{1}{2}(n+1)$. Since $d x_{n, i} / d t=c h$, we therefore note that

$$
D_{n, i}^{(n+1)}(t)=(c h)^{n+2} d^{(n+1)}\left(x_{n, i}(t)\right)
$$

and so

$$
D_{n, i}^{(n+1)}\left(\xi_{n, i}(t)\right)=O\left(h^{n+2}\right) .
$$

Thus, the integrals (4.3) and (4.4) are both $O\left(h^{n+2}\right)$ as required.

Using (4.1), we now obtain

$$
\begin{aligned}
\int_{x_{\ell}}^{x_{r}} d(x) d x & =\int_{t_{\ell}}^{t_{r}} D_{n, i}(t) d t \\
& =\int_{t_{\ell}}^{t_{r}} p_{n, i}(t) d t+O\left(h^{n+2}\right) \\
& =\int_{t_{\ell}}^{t_{r}} \sum_{k=0}^{n} M_{k} \ell_{n, k}(t) d t+O\left(h^{n+2}\right) \\
& =\sum_{k=0}^{n} m_{i+k+1} \int_{t_{\ell}}^{t_{r}} \ell_{n, k}(t) d t+O\left(h^{n+2}\right),
\end{aligned}
$$

where we used (2.6) in the final step. Clearly, in practice, we simply drop the error term when computing this integral.

4.2. Case II. The second type of integral that we will need to evaluate, when computing the Lyapunov exponent (see Section (7), is given by (2.5). As in the previous case, we again assume that the measures $m_{i}$ on the intervals $I_{i}, i=$ $1, \ldots, N$ are known exactly. Then we have that

$$
\int_{-1}^{1} F_{n, i}(t) D_{n, i}(t) d t=\int_{-1}^{1} F_{n, i}(t) p_{n, i}(t) d t+\int_{-1}^{1} F_{n, i}(t) e_{n, i}(t) d t .
$$

We first consider the error term in (4.5). For later use, we define the function

$$
\phi_{n+2}(t)=\prod_{j=0}^{n+1}\left(t-t_{j}\right)
$$

where the evenly spaced points $t_{j}$ are given by (2.3).

Theorem 4.2. If $n$ is odd, $F_{n, i} \in C^{2}[-1,1], D_{n, i} \in C^{n+2}[-1,1]$ and (1.1) is satisfied, then the error term in (4.5) is

$$
\int_{-1}^{1} F_{n, i}(t) e_{n, i}(t) d t=-\left[\frac{F_{n, i}^{\prime \prime}(\alpha) D_{n, i}^{(n+1)}(\delta)}{(n+2) !}+\frac{F_{n, i}^{\prime}(\alpha) D_{n, i}^{(n+2)}(\gamma)}{(n+3) !}\right] \int_{-1}^{1} t \phi_{n+2}(t) d t
$$

where $\alpha, \delta, \gamma \in(-1,1)$. 
If $n$ is even, $F_{n, i} \in C^{1}[-1,1], D_{n, i} \in C^{n+1}[-1,1]$ and (1.1) is satisfied, then the error term in (4.5) is

$$
\begin{aligned}
\int_{-1}^{1} F_{n, i}(t) e_{n, i}(t) d t=- & {\left[\frac{F_{n, i}^{\prime \prime}(\alpha) D_{n, i}^{(n+1)}(\delta)}{(n+2) !}+\frac{F_{n, i}^{\prime}(\alpha) D_{n, i}^{(n+2)}(\gamma)}{(n+3) !}\right] } \\
& \times(\alpha-1) \int_{-1}^{t_{n}} \phi_{n+2}(t) d t \\
& -\frac{F_{n, i}^{\prime}(\xi) D_{n, i}^{(n+1)}(\eta)}{(n+2) !} \int_{-1}^{1} \phi_{n+2}(t) d t
\end{aligned}
$$

where $\alpha, \delta, \gamma, \xi, \eta \in(-1,1)$.

Proof. We analyse the error by integrating by parts and using the error in the approximation to the measure $\nu_{n, i}(t)$ given by (2.13). Integrating by parts and using (2.16) gives

$$
\int_{-1}^{1} F_{n, i}(t) e_{n, i}(t) d t=\left[F_{n, i}(t) \varepsilon_{n+1, i}(t)\right]_{-1}^{1}-\int_{-1}^{1} F_{n, i}^{\prime}(t) \varepsilon_{n+1, i}(t) d t .
$$

The boundary terms vanish since $\varepsilon_{n+1, i}(-1)=0$ by (2.16) and $\varepsilon_{n+1, i}(1)=0$ by (2.16) and (2.14). Thus,

$$
\int_{-1}^{1} F_{n, i}(t) e_{n, i}(t) d t=-\frac{1}{(n+2) !} \int_{-1}^{1} F_{n, i}^{\prime}(t) \nu_{n, i}^{(n+2)}\left(\kappa_{n, i}(t)\right) \phi_{n+2}(t) d t .
$$

This error term is very similar to that for simple Newton-Cotes integration formulae [50] since the $t_{j}$ 's are evenly spaced points, except for the extra term $F_{n, i}^{\prime}(t)$ which complicates the analysis.

We first consider the case when $n$ is odd. In this case, $\phi_{n+2}(t)$ is an odd function since the points $t_{j}$ are evenly spaced, and this results in an error which is of higher order than would be expected, as in the case of Newton-Cotes formulae.

We define

$$
\psi_{n+3}(t)=\int_{-1}^{t} \phi_{n+2}(\tau) d \tau
$$

Since $\phi_{n+2}(t)$ is an odd function, it follows that $\psi_{n+3}(t)$ is of one sign [50], and that

$$
\psi_{n+3}(1)=0 .
$$

Integrating (4.8) by parts, the boundary terms again disappear using (4.9) and so $\int_{-1}^{1} F_{n, i}^{\prime}(t) \nu_{n, i}^{(n+2)}\left(\kappa_{n, i}(t)\right) \phi_{n+2}(t) d t=-\int_{-1}^{1} \frac{d}{d t}\left(F_{n, i}^{\prime}(t) \nu_{n, i}^{(n+2)}\left(\kappa_{n, i}(t)\right)\right) \psi_{n+3}(t) d t$.

Since $\psi_{n+3}(t)$ is of one sign, the Mean Value Theorem for Integrals can be used, giving

$$
\begin{aligned}
\int_{-1}^{1} & F_{n, i}^{\prime}(t) \nu_{n, i}^{(n+2)}\left(\kappa_{n, i}(t)\right) \phi_{n+2}(t) d t \\
& =-\left.\frac{d}{d t}\left(F_{n, i}^{\prime}(t) \nu_{n, i}^{(n+2)}\left(\kappa_{n, i}(t)\right)\right)\right|_{t=\alpha} \int_{-1}^{1} \psi_{n+3}(t) d t \\
& =\left.\frac{d}{d t}\left(F_{n, i}^{\prime}(t) \nu_{n, i}^{(n+2)}\left(\kappa_{n, i}(t)\right)\right)\right|_{t=\alpha} \int_{-1}^{1} t \phi_{n+2}(t) d t
\end{aligned}
$$


where $\alpha \in(-1,1)$ and the second step involved another integration by parts.

It can be shown [49] that

$$
\frac{d}{d t} \nu_{n, i}^{(n+2)}\left(\kappa_{n, i}(t)\right)=\frac{\nu_{n, i}^{(n+3)}\left(\beta_{n, i}(t)\right)}{n+3},
$$

for some $\beta_{n, i}(t) \in(-1,1)$. Thus, substituting (4.10) into (4.8) and using (4.11), we obtain

$$
\int_{-1}^{1} F_{n, i}(t) e_{n, i}(t) d t=-\left[\frac{F_{n, i}^{\prime \prime}(\alpha) \nu_{n, i}^{(n+2)}(\delta)}{(n+2) !}+\frac{F_{n, i}^{\prime}(\alpha) \nu_{n, i}^{(n+3)}(\gamma)}{(n+3) !}\right] \int_{-1}^{1} t \phi_{n+2}(t) d t,
$$

where $\delta=\kappa_{n, i}(\alpha)$ and $\gamma=\beta_{n, i}(\alpha)$. Now $\nu_{n, i}^{\prime}(t)=D_{n, i}(t)$ and so substituting for $\nu_{n, i}$ gives (4.6) as claimed.

When $n$ is even, the proof is more complicated, as it is for the standard NewtonCotes result. We first break the integral in (4.8) into two, giving

$$
\begin{aligned}
\int_{-1}^{1} F_{n, i}^{\prime}(t) \nu_{n, i}^{(n+2)}\left(\kappa_{n, i}(t)\right) \phi_{n+2}(t) d t \\
=\int_{-1}^{t_{n}} F_{n, i}^{\prime}(t) \nu_{n, i}^{(n+2)}\left(\kappa_{n, i}(t)\right) \phi_{n+2}(t) d t \\
\quad+\int_{t_{n}}^{1} F_{n, i}^{\prime}(t) \nu_{n, i}^{(n+2)}\left(\kappa_{n, i}(t)\right) \phi_{n+2}(t) d t
\end{aligned}
$$

For the second integral, $\phi_{n+2}(t)$ is of one sign on the interval $\left[t_{n}, 1\right]$ and so the Mean Value Theorem for Integrals gives that

$$
\int_{t_{n}}^{1} F_{n, i}^{\prime}(t) \nu_{n, i}^{(n+2)}\left(\kappa_{n, i}(t)\right) \phi_{n+2}(t) d t=F_{n, i}^{\prime}(\theta) \nu_{n, i}^{(n+2)}\left(\kappa_{n, i}(\theta)\right) \int_{t_{n}}^{1} \phi_{n+2}(t) d t
$$

for some $\theta \in\left(t_{n}, 1\right)$.

We rewrite the first integral in (4.12) as

$$
\int_{-1}^{t_{n}} F_{n, i}^{\prime}(t) \nu_{n, i}^{(n+2)}\left(\kappa_{n, i}(t)\right) \phi_{n+2}(t) d t=\int_{-1}^{t_{n}} F_{n, i}^{\prime}(t) \nu_{n, i}^{(n+2)}\left(\kappa_{n, i}(t)\right)(t-1) \phi_{n+1}(t) d t,
$$

and we note that $\phi_{n+1}(t)$ is an odd function about the midpoint of the interval $\left[-1, t_{n}\right]$. Thus, using the result (4.10) for $n$ odd then gives

$$
\begin{aligned}
& \int_{-1}^{t_{n}} F_{n, i}^{\prime}(t) \nu_{n, i}^{(n+2)}\left(\kappa_{n, i}(t)\right) \phi_{n+2}(t) d t \\
&=\left.\frac{d}{d t}\left\{F_{n, i}^{\prime}(t) \nu_{n, i}^{(n+2)}\left(\kappa_{n, i}(t)\right)(t-1)\right\}\right|_{t=\alpha} \\
& \quad \times \int_{-1}^{t_{n}} t \phi_{n+1}(t) d t \\
&=\left[\left.\frac{d}{d t}\left\{F_{n, i}^{\prime}(t) \nu_{n, i}^{(n+2)}\left(\kappa_{n, i}(t)\right)\right\}\right|_{t=\alpha}(\alpha-1)\right. \\
&\left.+F_{n, i}^{\prime}(\alpha) \nu_{n, i}^{(n+2)}\left(\kappa_{n, i}(\alpha)\right)\right] \int_{-1}^{t_{n}} t \phi_{n+1}(t) d t
\end{aligned}
$$


where $\alpha \in\left(-1, t_{n}\right)$. Now since $\phi_{n+1}(t)$ is odd about the midpoint of the interval,

$$
\int_{-1}^{t_{n}} t \phi_{n+1}(t) d t=\int_{-1}^{t_{n}}(t-1) \phi_{n+1}(t) d t=\int_{-1}^{t_{n}} \phi_{n+2}(t) d t .
$$

Combining the integrals (4.13) and (4.14), and using (4.15), we therefore obtain

$$
\begin{aligned}
\int_{-1}^{1} F_{n, i}^{\prime}(t) \nu_{n, i}^{(n+2)}\left(\kappa_{n, i}(t)\right) \phi_{n+2}(t) d t= & {\left[\left.\frac{d}{d t}\left\{F_{n, i}^{\prime}(t) \nu_{n, i}^{(n+2)}\left(\kappa_{n, i}(t)\right)\right\}\right|_{t=\alpha}(\alpha-1)\right.} \\
& \left.+F_{n, i}^{\prime}(\alpha) \nu_{n, i}^{(n+2)}\left(\kappa_{n, i}(\alpha)\right)\right] \int_{-1}^{t_{n}} \phi_{n+2}(t) d t \\
& +F_{n, i}^{\prime}(\theta) \nu_{n, i}^{(n+2)}\left(\kappa_{n, i}(\theta)\right) \int_{t_{n}}^{1} \phi_{n+2}(t) d t
\end{aligned}
$$

Now the two integrals have the same sign 52 and so

$$
\begin{aligned}
\int_{-1}^{1} F_{n, i}^{\prime}(t) \nu_{n, i}^{(n+2)}\left(\kappa_{n, i}(t)\right) \phi_{n+2}(t) d t= & \left.\frac{d}{d t}\left\{F_{n, i}^{\prime}(t) \nu_{n, i}^{(n+2)}\left(\kappa_{n, i}(t)\right)\right\}\right|_{t=\alpha} \\
& \times(\alpha-1) \int_{-1}^{t_{n}} \phi_{n+2}(t) d t \\
& +F_{n, i}^{\prime}(\xi) \nu_{n, i}^{(n+2)}\left(\kappa_{n, i}(\xi)\right) \\
& \times\left[\int_{-1}^{t_{n}} \phi_{n+2}(t) d t+\int_{t_{n}}^{1} \phi_{n+2}(t) d t\right] \\
= & \left.\frac{d}{d t}\left\{F_{n, i}^{\prime}(t) \nu_{n, i}^{(n+2)}\left(\kappa_{n, i}(t)\right)\right\}\right|_{t=\alpha} \\
& \times(\alpha-1) \int_{-1}^{t_{n}} \phi_{n+2}(t) d t \\
& +F_{n, i}^{\prime}(\xi) \nu_{n, i}^{(n+2)}\left(\kappa_{n, i}(\xi)\right) \int_{-1}^{1} \phi_{n+2}(t) d t
\end{aligned}
$$

for some $\xi \in(-1,1)$ (and satisfying $\alpha \leq \xi \leq \theta)$. Expanding the derivative term and using (4.11) gives

$$
\begin{aligned}
\int_{-1}^{1} F_{n, i}^{\prime}(t) \nu_{n, i}^{(n+2)}\left(\kappa_{n, i}(t)\right) \phi_{n+2}(t) d t \\
=\left[F_{n, i}^{\prime \prime}(\alpha) \nu_{n, i}^{(n+2)}\left(\kappa_{n, i}(\alpha)\right)+\frac{F_{n, i}^{\prime}(\alpha) \nu_{n, i}^{(n+3)}\left(\beta_{n, i}(\alpha)\right)}{n+3}\right] \\
\quad \times(\alpha-1) \int_{-1}^{t_{n}} \phi_{n+2}(t) d t \\
\quad+F_{n, i}^{\prime}(\xi) \nu_{n, i}^{(n+2)}\left(\kappa_{n, i}(\xi)\right) \int_{-1}^{1} \phi_{n+2}(t) d t
\end{aligned}
$$


where $\beta_{n, i}(\alpha) \in(-1,1)$. Substituting back into (4.8) then gives

$$
\begin{aligned}
\int_{-1}^{1} F_{n, i}(t) e_{n, i}(t) d t= & -\left[\frac{F_{n, i}^{\prime \prime}(\alpha) \nu_{n, i}^{(n+2)}\left(\kappa_{n, i}(\alpha)\right)}{(n+2) !}+\frac{F_{n, i}^{\prime}(\alpha) \nu_{n, i}^{(n+3)}\left(\beta_{n, i}(\alpha)\right)}{(n+3) !}\right] \\
& \times(\alpha-1) \int_{-1}^{t_{n}} \phi_{n+2}(t) d t \\
& -\frac{F_{n, i}^{\prime}(\xi) \nu_{n, i}^{(n+2)}\left(\kappa_{n, i}(\xi)\right)}{(n+2) !} \int_{-1}^{1} \phi_{n+2}(t) d t .
\end{aligned}
$$

Finally, substituting for $\nu_{n, i}$ using $\nu_{n, i}^{\prime}(t)=D_{n, i}(t)$ gives the stated result, with $\delta=\kappa_{n, i}(\alpha), \gamma=\beta_{n, i}(\alpha)$ and $\eta=\kappa_{n, i}(\xi)$.

These results can be extended to the case of evaluating an integral over the whole interval $I$.

Theorem 4.3. Let $p_{N}^{n}(x)$ be the discontinuous piecewise polynomial approximation to the density, assuming that the measures $m_{i}$ on the intervals $I_{i}, i=1, \ldots, N$ are known exactly, i.e., (1.1) holds.

If $n$ is odd, $f \in C^{2}(I), d \in C^{n+2}(I)$ and $f(x)$ is not a constant, then

$$
\int_{I} f(x) d(x) d x=\int_{I} f(x) p_{N}^{n}(x) d x+O\left(h^{n+3}\right) .
$$

If $n$ is even, $f \in C^{1}(I), d \in C^{n+1}(I)$ and $f(x)$ is not a constant, then

$$
\int_{I} f(x) d(x) d x=\int_{I} f(x) p_{N}^{n}(x) d x+O\left(h^{n+2}\right) .
$$

Proof. We first note that if $f(x)$ is a constant, then the error term in both cases is zero by construction, and hence this case has been excluded.

The discontinuous piecewise polynomial approximation $p_{N}^{n}$ comprises $N /(n+1)$ separate polynomials $\tilde{p}_{n, i} \in \Pi_{n}$ which are defined by

$$
\tilde{p}_{n, i}(x)=\left.p_{N}^{n}(x)\right|_{\left[x_{i}, x_{i+n+1}\right]}, \quad i=0, n+1,2(n+1), \ldots, N-(n+1) .
$$

Converting these to polynomials in the variable $t$ we define

$$
p_{n, i}(t)=\tilde{p}_{n, i}\left(x_{n, i}(t)\right), \quad i=0, n+1,2(n+1), \ldots, N-(n+1),
$$

where $x_{n, i}(t)$ is given in (2.2).

The results of Theorem 4.2 can be converted back to the original $x$ coordinates in order to obtain error terms as a power of $h$. As in the proof of Theorem 4.1, we note that $D_{n, i}(t)=\operatorname{ch} d\left(x_{n, i}(t)\right)$ and that $d x_{n, i} / d t=c h$, where $c=\frac{1}{2}(n+1)$. Using these results, we obtain, for $n$ odd,

$$
\begin{aligned}
\int_{x_{i}}^{x_{i+n+1}} f(x) d(x) d x & =\int_{-1}^{1} F_{n, i}(t) D_{n, i}(t) d t \\
& =\int_{-1}^{1} F_{n, i}(t) p_{n, i}(t) d t+\int_{-1}^{1} F_{n, i}(t) e_{n, i}(t) d t \\
& =\int_{-1}^{1} F_{n, i}(t) p_{n, i}(t) d t+O\left(h^{n+4}\right) .
\end{aligned}
$$


Similarly, for $n$ even, we have

$$
\int_{x_{i}}^{x_{i+n+1}} f(x) d(x) d x=\int_{-1}^{1} F_{n, i}(t) p_{n, i}(t) d t+O\left(h^{n+3}\right) .
$$

We note that in the first term of (4.7), the term $\alpha-1$ can be expressed, using (2.1), as

$$
\alpha-1=-2+\frac{2\left(\tilde{\alpha}-x_{i}\right)}{(n+1) h}=\frac{2\left(\tilde{\alpha}-x_{i+n+1}\right)}{(n+1) h}=O(1 / h),
$$

where $\tilde{\alpha}=x_{n, i}(\alpha) \in\left(x_{i}, x_{i+n+1}\right)$, and so both terms in (4.7) are $O\left(h^{n+3}\right)$.

Integrating over the whole interval $I$ requires the sum of $N /(n+1)$ such integrals. As usual, the error term for such a composite quadrature rule is one power of $h$ less than for the simple rule as it involves the sum of the errors over the $N /(n+1)$ intervals, and $N=O\left(h^{-1}\right)$. This gives the stated results.

Theorem 4.4. The results of Theorem 4.3 hold if $f \in C^{0}(I)$ but is piecewise $C^{2}$ ( $n$ odd) or piecewise $C^{1}$ ( $n$ even).

Proof. Clearly for intervals $I_{i}$ which do not contain a discontinuity in the derivative of $f(x)$, Theorem 4.2 still holds. Thus, we need consider only those intervals where there is point of discontinuity of the derivative. In particular, we will consider only the case where there is a single point of discontinuity in any particular interval, but the results can easily be generalised to the case of multiple points of discontinuity. Thus, we assume that on some interval $I_{i}$, the function $F_{n, i}(t)=f\left(x_{n, i}(t)\right)$ is given by

$$
F_{n, i}(t)= \begin{cases}F_{1}(t), & -1 \leq t \leq t^{*} \\ F_{2}(t), & t^{*} \leq t \leq 1\end{cases}
$$

where $F_{1}\left(t^{*}\right)=F_{2}\left(t^{*}\right)$, but $F_{1}^{\prime}\left(t^{*}\right) \neq F_{2}^{\prime}\left(t^{*}\right)$. The result obtained from the first integration by parts in the proof of Theorem 4.2 still holds since $F$ is continuous. However, the resulting integral must be split into two and so we have

$$
\begin{aligned}
\int_{-1}^{1} F_{n, i}(t) e_{n, i}(t) d t=-\frac{1}{(n+2) !} & {\left[\int_{-1}^{t^{*}} F_{1}^{\prime}(t) \nu_{n, i}^{(n+2)}\left(\kappa_{n, i}(t)\right) \phi_{n+2}(t) d t\right.} \\
& \left.+\int_{t^{*}}^{1} F_{2}^{\prime}(t) \nu_{n, i}^{(n+2)}\left(\kappa_{n, i}(t)\right) \phi_{n+2}(t) d t\right] .
\end{aligned}
$$

For $n$ odd, the next step in the proof of Theorem 4.2 was to integrate by parts again, with the boundary terms vanishing. However, in this case, the boundary terms do not vanish, but integration by parts gives

$$
\begin{aligned}
\int_{-1}^{1} F_{n, i}(t) e_{n, i}(t) d t= & -\frac{1}{(n+2) !}\left(F_{1}^{\prime}\left(t^{*}\right)-F_{2}^{\prime}\left(t^{*}\right)\right) \nu_{n, i}^{(n+2)}\left(\kappa_{n, i}\left(t^{*}\right)\right) \psi_{n+3}\left(t^{*}\right) \\
& +\frac{1}{(n+2) !}\left[\int_{-1}^{t^{*}} \frac{d}{d t}\left(F_{1}^{\prime}(t) \nu_{n, i}^{(n+2)}\left(\kappa_{n, i}(t)\right)\right) \psi_{n+3}(t) d t\right. \\
& \left.+\int_{t^{*}}^{1} \frac{d}{d t}\left(F_{2}^{\prime}(t) \nu_{n, i}^{(n+2)}\left(\kappa_{n, i}(t)\right)\right) \psi_{n+3}(t) d t\right]
\end{aligned}
$$

Now $\psi_{n+3}(t)$ is of one sign [50] and so, by the Mean Value Theorem for Integrals, the derivative term can be taken outside of each integral, giving two terms, each 
TABLE 2. The order of the error term in (4.16) and (4.17) for different values of $n$.

\begin{tabular}{|c|c|}
\hline$n$ & Error Term \\
\hline 0 & $O\left(h^{2}\right)$ \\
1 & $O\left(h^{4}\right)$ \\
2 & $O\left(h^{4}\right)$ \\
3 & $O\left(h^{6}\right)$ \\
4 & $O\left(h^{6}\right)$ \\
\hline
\end{tabular}

of which are similar to those obtained in Theorem 4.2. Converting back to $x$ coordinates, as in the proof of Theorem 4.3 we again find that the integral terms are $O\left(h^{n+4}\right)$. However, the boundary terms, which were not present previously, are $O\left(h^{n+3}\right)$. Now when we sum over all the intervals, we sum the integrals and this sum gives an error of $O\left(h^{n+3}\right)$ since $N=O\left(h^{-1}\right)$, as previously. In the case of the boundary terms, we note that only intervals containing a discontinuity of the derivative of $f$ have such boundary terms, and this is a fixed number which does not depend on $h$. Thus, the contribution of these boundary terms is also $O\left(h^{n+3}\right)$ and so the total error is $O\left(h^{n+3}\right)$ as previously.

A similar analysis applies for $n$ even.

A summary of the order of errors for different values of $n$ is given in Table 2 , which shows a similar pattern to the error in Newton-Cotes integration.

4.3. Gaussian quadrature. In Case I, the integral on the right-hand side of (4.1) is that of a polynomial, and so can be evaluated exactly. However, in Case II and for a general function $F_{n, i}(t)$, the first integral on the right-hand side of (4.5) may have to be evaluated numerically using Gaussian quadrature. When integrating a function $G(t)$ on the interval $[-1,1]$ using an $m$ point Gaussian quadrature rule, the error term is given by

$$
\frac{G^{(2 m)}(\eta)}{(2 m) !} \int_{-1}^{1}\left[P_{m}(t)\right]^{2} d t
$$

where $P_{m}(t)$ is the $m$ th Legendre polynomial and $\eta \in(-1,1)$ 7]. Converting back to the original $x$ coordinates, we note again that each derivative of a function introduces a power of $h$, since $d x_{n, i} / d t=\frac{1}{2}(n+1) h$. The functions we integrate always involve the density function $D_{n, i}(t)$ and the change of function from $D_{n, i}(t)$ (which is approximated by $\left.p_{n, i}(t)\right)$ back to $d(x)$ introduces another power of $h$. Thus, the error associated with a simple $m$ point Gaussian quadrature rule is $O\left(h^{2 m+1}\right)$.

In Case II, as shown in the proof of Theorem 4.3, the approximation error for a single integral for $n$ odd is $O\left(h^{n+4}\right)$. Thus, the error from the quadrature will be the same order as the approximation error if $m=(n+3) / 2$. Similarly, for $n$ even, the approximation error for a single integral is $O\left(h^{n+3}\right)$ and so the quadrature error will be the same order if $m=(n+2) / 2$. Thus, for $n=2 k-1$ or $n=2 k$, the number of integration points used should be at least $m=k+1$ to ensure that the error in the integration does not dominate the approximation error. 


\section{Convergence Analysis}

We now consider convergence of the method for computing the invariant density by discretising the Frobenius-Perron equation as described in Section 3 as $N \rightarrow \infty$. We define the approximation space

$$
\Delta_{N}^{n}=\left\{f:[0,1] \rightarrow[0,1]:\left.f\right|_{\left[x_{i}, x_{i+n+1}\right]} \in \Pi_{n}, i=0, n+1,2(n+1), \ldots, N-(n+1)\right\},
$$

of functions which are piecewise polynomials of degree $n$ on $n+1$ adjacent intervals from the given partition. Note that $\operatorname{dim}\left(\Delta_{N}^{n}\right)=N$ and functions in $\Delta_{N}^{n}$ are not necessarily continuous.

In Section 2, for a given density $d$, we constructed a function $p_{N}^{n} \in \Delta_{N}^{n}$ which satisfies

$$
\int_{I_{i}} p_{N}^{n}(x) d x=m_{i}=\int_{I_{i}} d(x) d x, \quad i=1, \ldots, N .
$$

In other words, $p_{N}^{n}=L_{N}^{n} d$, where the projection $L_{N}^{n}: L^{1}([0,1], \mathbb{R}) \rightarrow \Delta_{N}^{n}$ is characterised by

$$
\int_{I_{i}}\left(L_{N}^{n} d\right)(x) d x=\int_{I_{i}} d(x) d x, \quad i=1, \ldots, N,
$$

for $d \in L^{1}([0,1], \mathbb{R})$. Let $($,$) denote the duality pair between L^{1}([0,1], \mathbb{R})$ and its dual and let $T_{N}$ be the space spanned by the characteristic functions $\chi_{1}, \ldots, \chi_{N}$ on the intervals $I_{1}, \ldots, I_{N}$. Then (5.1) can alternatively be written as

$$
\left(L_{N}^{n} d, v\right)=(d, v) \text { for all } v \in T_{N} .
$$

Thus, $L_{N}^{n} d$ is the Petrov-Galerkin projection of $d$ (with respect to the spaces $\Delta_{N}^{n}$ and $\left.T_{N}\right)$.

In Section 3 , for a given map $g:[0,1] \rightarrow[0,1]$, we constructed an approximate invariant density $\tilde{d} \in \Delta_{N}^{n}$ by requiring that

$$
\int_{I_{i}} \tilde{d}(x) d x=\int_{g^{-1}\left(I_{i}\right)} \tilde{d}(x) d x, \quad i=1, \ldots, N .
$$

The next lemma shows that this is equivalent to computing $\tilde{d} \in \Delta_{N}^{n}$ such that

$$
L_{N}^{n} P \tilde{d}=\tilde{d}
$$

where $P$ is the Frobenius-Perron operator, i.e., $\tilde{d}$ is a fixed point of the discretised Frobenius-Perron operator

$$
P_{N}^{n}:=L_{N}^{n} P
$$

Lemma 5.1. A function $\tilde{d} \in \Delta_{N}^{n}$ satisfies (5.3) if and only if it is a fixed point of the discrete Frobenius-Perron operator $P_{N}^{n}$.

Proof. If $\tilde{d}$ satisfies (5.3) then $(\tilde{d}, v)=(P \tilde{d}, v)$ for all $v \in T_{N}$ since the characteristic functions on the intervals $I_{i}$ are a basis of $T_{N}$. By the definition of the projection $L_{N}^{n}$ in (5.2), $\left(L_{N}^{n} P \tilde{d}, v\right)=(P \tilde{d}, v)$ for all $v \in T_{N}$. Combining these two equalities, we arrive at

$$
\left(L_{N}^{n} P \tilde{d}, v\right)=(\tilde{d}, v) \quad \text { for all } v \in T_{N},
$$

and since $\tilde{d} \in \Delta_{N}^{n}$ and the projection is unique, it follows that $P_{N}^{n} \tilde{d}=\tilde{d}$. 
To prove the converse, let $\tilde{d} \in \Delta_{N}^{n}$ be a fixed point of $P_{N}^{n}$, then $\left(L_{N}^{n} P \tilde{d}, v\right)=(\tilde{d}, v)$ for all $v \in T_{N}$. From the definition of $L_{N}^{n},\left(L_{N}^{n} P \tilde{d}, v\right)=(P \tilde{d}, v)$ for all $v \in T_{N}$. Combining these two equalities, we obtain

$$
(P \tilde{d}, v)=(\tilde{d}, v) \text { for all } v \in T_{N},
$$

which is equivalent to (5.3).

Analogous to the proof of Lemma 8 in [17] we can show:

Lemma 5.2. The discretised Frobenius-Perron operator $P_{N}^{n}$ has a nonzero fixed point

which satisfies

$$
P_{N}^{n} d_{N}^{n}=d_{N}^{n} \in \Delta_{N}^{n}
$$

$$
\int_{0}^{1} d_{N}^{n}(x) d x=1
$$

We note that the solution $d_{N}^{n}$ of the discretised Frobenius-Perron equation is not the same as $p_{N}^{n}=L_{N}^{n} d$, which is the projection of the true density $d$ onto the approximation space.

We use the framework of [17] in order to prove convergence of our scheme in the case that $g$ is piecewise $C^{2}[0,1]$ and stretching (i.e., $\inf _{x \in[0,1]}\left|g^{\prime}(x)\right|>1$ ). The only difference to the setup in [17] is that we are dealing with a Petrov-Galerkin projection instead of a standard Galerkin projection. We are thus working with a fixed conjugate basis $\left\{A^{i}\right\}_{0}^{n}$ of $\Delta_{n+1}^{n}$,

$$
A^{i}=\chi_{i+1}, \quad i=0, \ldots, n,
$$

and choose the basis $\left\{a^{i}\right\}_{0}^{n}$ of $\Delta_{n+1}^{n}$ such that

$$
\left(a^{i}, A^{k}\right)=\delta_{i k}, \quad i, k=0, \ldots, n .
$$

Lemma 10 of [17] now yields stability of the projection $L_{N}^{n}$ :

Lemma 5.3. There exist constants $\gamma_{1}(n)$ and $\gamma_{2}(n)$ such that for $d \in L^{1}(0,1)$,

$$
\left\|L_{N}^{n} d\right\|_{1} \leq \gamma_{1}(n)\|d\|_{1},
$$

and if $d \in B V(0,1)$ then

$$
\bigvee_{0}^{1} L_{N}^{n} d \leq \gamma_{2}(n) \bigvee_{0}^{1} d
$$

As in [17, consistency of the projection is a standard result from approximation theory:

Lemma 5.4. Let $d \in L^{1}(0,1)$, then

$$
\lim _{N \rightarrow \infty}\left\|d-L_{N}^{n} d\right\|_{1}=0 .
$$

If $g$ is piecewise $C^{2}[0,1]$ and stretching, then the Lasota-Yorke theorem [43] implies that for any function $d \in L^{1}(0,1)$ of bounded variation, $P d$ is of bounded variation as well and there exist constants $\alpha>0$ and $\beta \leq 2 / M$ (with $M=$ $\left.\inf _{x \in[0,1]}\left|g^{\prime}(x)\right|>1\right)$ such that

$$
\bigvee_{0}^{1} P d \leq \alpha\|d\|_{1}+\beta \bigvee_{0}^{1} d
$$


Corollary 3 and 4 of [17] now yield convergence of our scheme:

Theorem 5.5. Let $g$ be piecewise $C^{2}[0,1]$ and stretching. If $\beta<1$ and $\gamma_{2}(n) \beta<1$ then the sequence $\left(d_{N}^{n}\right)_{N}$ of fixed points of the discretised Frobenius-Perron operator has a subsequence which converges in $L^{1}(0,1)$ to an invariant density $d$ of $g$. Moreover,

$$
\left\|d-d_{N}^{n}\right\|_{1}=O\left(\left\|d-L_{N}^{n} d\right\|_{1}\right) \quad \text { as } N \rightarrow \infty .
$$

Corollary 5.6. Under the conditions of Theorem 5.5 and for a given value of $n$,

$$
\left\|d-d_{N}^{n}\right\|_{1}=O\left(h^{n+1}\right)=O\left(\frac{1}{N^{n+1}}\right) \quad \text { as } N \rightarrow \infty .
$$

Proof. The function $L_{N}^{n} d=p_{N}^{n}$ is the piecewise polynomial approximation to $d$ assuming that the measures $m_{i}$ on the intervals $I_{i}, i=1, \ldots, n$ are known exactly. This is precisely the situation that we considered in Theorem 4.1 Thus,

$$
\begin{aligned}
\left\|d-L_{N}^{n} d\right\|_{1} & =\int_{I}\left|d(x)-\left(L_{N}^{n} d\right)(x)\right| d x \\
& =\sum_{i=1}^{N} \int_{I_{i}}\left|d(x)-\left(L_{N}^{n} d\right)(x)\right| d x \\
& =\sum_{i=1}^{N} \int_{t_{\ell}}^{t_{r}}\left|D_{i}(t)-p_{n, i}(t)\right| d t,
\end{aligned}
$$

for appropriate values of $t_{\ell}$ and $t_{r}$ and where $D_{i}(t)$ and $p_{n, i}(t)$ are the restrictions of $d(x)$ and $L_{N}^{n} d(x)$ respectively to the interval $I_{i}$. By (4.2), the final integral above is $O\left(h^{n+2}\right)$ and since a sum of $N=O\left(h^{-1}\right)$ such terms is required, then

$$
\left\|d-L_{N}^{n} d\right\|_{1}=O\left(h^{n+1}\right) .
$$

Finally, Theorem 5.5 tells us that

$$
\left\|d-d_{N}^{n}\right\|_{1}=O\left(\left\|d-L_{N}^{n} d\right\|_{1}\right)=O\left(h^{n+1}\right)=O\left(\frac{1}{N^{n+1}}\right),
$$

as required.

Remark 5.7. Theorem 5.5 requires the map $g$ to be stretching, i.e., $\inf _{x \in[0,1]}\left|g^{\prime}(x)\right|>$ 1 as this is a condition of the Lasota-Yorke Theorem 43 . However, by Theorem 3 of 43, this condition can be relaxed by requiring that $g^{m}$ be stretching for some positive integer $m$ and that $\inf _{x \in[0,1]}\left|g^{\prime}(x)\right|>0$. Under these conditions, Theorem 5.5 also holds.

\section{EXAmples}

6.1. Example 1. As an example of the preceding theory, we consider in detail the map $g_{1}:[0,1] \rightarrow[0,1]$ defined by

$$
g_{1}(x)= \begin{cases}\frac{2 x}{1-x^{2}}, & 0 \leq x \leq \sqrt{2}-1, \\ \frac{1-x^{2}}{2 x}, & \sqrt{2}-1 \leq x \leq 1 .\end{cases}
$$

The invariant density for this map (which is the map $S_{3}$ of [17]) is

$$
d_{1}(x)=\frac{4}{\pi\left(1+x^{2}\right)} .
$$



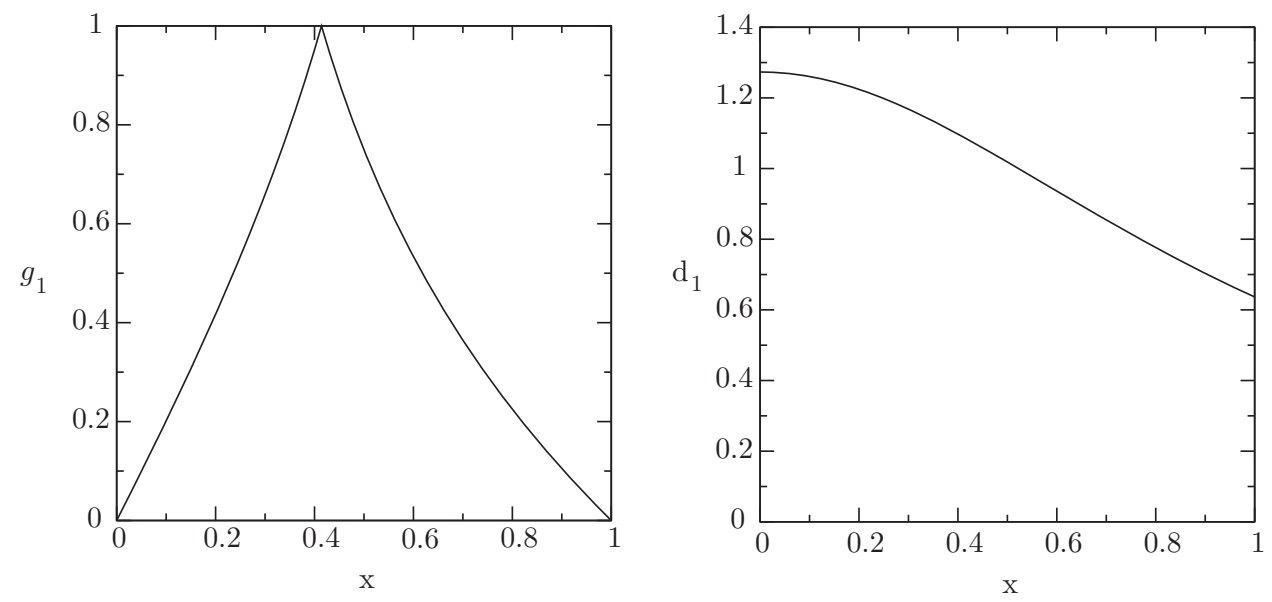

Figure 2. The map $g_{1}$ defined by (6.1) and its density function $d_{1}$.

The function $g_{1}$ and the density $d_{1}$ are shown in Figure 2 .

We note that $g_{1}$ is piecewise $C^{2}[0,1]$, but is not stretching as $g_{1}^{\prime}(1)=-1$. However, $g_{1}^{2}$ is stretching and $\inf _{x \in[0,1]}\left|g_{1}^{\prime}(x)\right|=1$ and so the conditions of Remark 5.7 are satisfied.

We divided the interval $[0,1]$ into $N$ subintervals of width $h=1 / N$ and took groups of $n+1$ subintervals to construct a measure-preserving polynomial approximation to the density on these intervals. The method described in the previous section was used to compute the matrix representation $A$ of the Frobenius-Perron operator and the eigenvector $\tilde{\mathbf{m}}$ of $A$ associated with the eigenvalue nearest to +1 was computed using the inverse power method [7]. Since the true density function is known for this example, the one norm of the difference between the exact function $d_{1}(x)$ and the piecewise polynomial approximation $d_{N}^{n}(x)$ was determined. The program was written in MAPLE so that the accuracy of the calculations could be increased if required. The results are shown in Figure 3. We note that the slope of the final segment of each of these lines is given for $n=0,1,2,3$ by -1.035 , $-2.049,-3.049$ and -3.917 , respectively, which gives experimental verification of the theoretical result of Corollary 5.6 that the rate of convergence is $O\left(h^{n+1}\right)$.

To illustrate the power of this method, taking 16 subintervals with groups of 4 subintervals used to compute four cubic polynomial approximations to the density gives an error of

$$
\left\|d_{1}(x)-d_{16}^{3}(x)\right\|_{1}=8.776219 \times 10^{-6} .
$$

Thus, by using higher order polynomials, very accurate results can be obtained using only a small number of subintervals. The standard Ulam method using only piecewise constant approximations to the density and the same number of intervals gives an error of

$$
\left\|d_{1}(x)-d_{16}^{0}(x)\right\|_{1}=1.168727 \times 10^{-2} .
$$

For piecewise constant approximations, doubling $N$ approximately halves the error, as can be seen in Figure 3. Taking the error for $N=128$ and successively halving it, we obtain an approximation to the error for $N=128 \times 2^{7}=16,384$ of $1.307149 \times$ 


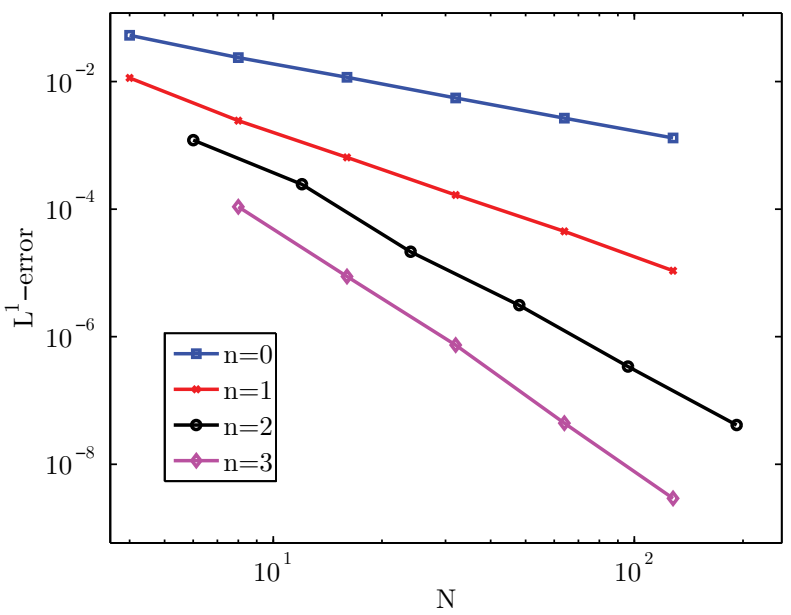

Figure 3. $L^{1}$-errors in computed invariant densities for different values of $n$ and $N$ for the map $g_{1}$.

$10^{-3} / 2^{7}=1.02121 \times 10^{-5}$, which is still slightly larger than the error above using $p_{16}^{3}(x)$ !

We note that the results of [17] for this map appear to show convergence rates of $O(h), O\left(h^{2}\right)$ and $O\left(h^{2}\right)$ for the piecewise constant, linear and quadratic cases. We get the same rates for the constant and linear cases, but we have $O\left(h^{3}\right)$ for the piecewise quadratic case.

6.2. Example 2. As another example, we have applied our method to the map

$$
g_{2}(x)= \begin{cases}\frac{2 x}{1-x}, & 0 \leq x \leq \frac{1}{3}, \\ \frac{1-x}{2 x}, & \frac{1}{3} \leq x \leq 1,\end{cases}
$$

which is the map $S_{4}$ of [17]. This map has invariant density given by

$$
d_{2}(x)=\frac{2}{(1+x)^{2}} \text {. }
$$

Our results for this map are similar to those for the previous map and show the same rates of convergence.

6.3. Example 3. We also consider the map

$$
g_{3}(x)=\left(\frac{1}{8}-2\left|x-\frac{1}{2}\right|^{3}\right)^{1 / 3}+\frac{1}{2}
$$

which is the map $S_{2}$ of [17]. In this case, the invariant density is

$$
d_{3}(x)=12\left(x-\frac{1}{2}\right)^{2},
$$

which is quadratic. From the proof of Theorem 4.1, it is clear that the error term for the integrals involved in setting up the Frobenius-Perron operator depend on the $(n+1)$ th derivative of the density. Since the density is a quadratic function in this 
case, we would expect the error to be zero with a piecewise quadratic approximation and this is indeed the case. This is in contrast to the results of [17, whose results appear to show $O\left(h^{3}\right)$ convergence with piecewise quadratic approximations for this map. We again achieve convergence rates of $O(h)$ and $O\left(h^{2}\right)$ for piecewise constant and linear approximations.

The interesting aspect of this example is that the function $g_{3}$ does not fulfill the conditions of Theorem 5.5, as it is neither $C^{2}[0,1]$ (as $g_{3}^{\prime}(x)=\infty$ at the two points $x=\frac{1}{2} \pm 2^{-4 / 3}$ ) nor stretching (since the first and second derivatives at $x=1 / 2$ are zero). Also, no iterates of $g_{3}$ are stretching and so Remark 5.7 is of no help either in this case. Thus, it seems that the method converges for a wider class of maps than those specified in Theorem 5.5] and Remark 5.7. In fact, recently, much progress has been made in establishing the existence of invariant densities for general interval maps which are not expanding; see for example [1, 5, 6]. However, it remains to be explored whether the theory developed in these papers yields the tools in order to prove Ulam's conjecture or the convergence of the scheme developed in this paper.

6.4. Example 4. All of the previous examples we have considered have had a known density, which was useful for comparing our theoretical predictions with numerical examples. However, our method also works for problems where the density is not known. To illustrate this, we consider the double standard map [46] which is defined by

$$
g_{4}(x)=2 x+a+\frac{b}{\pi} \sin (2 \pi x) \quad \bmod 1 .
$$

We choose parameter values $a=b=0.7$. For these values, the map is not stretching, but the third iterate is stretching and so, by Remark 5.7. Theorem 5.5 can still be applied. We have also assumed that the invariant density is smooth. It is known that this map is semi-conjugate to the doubling map via a continuous, increasing function [46. If this function is also smooth, then the density of the map (6.3) will be smooth, but this has not been proved to our knowledge. However, we will assume that this is the case and will apply our method to this problem.

The function $g_{4}$ and the computed density, obtained using a piecewise polynomial approximation comprising 16 cubic polynomials and a total of $N=64$ subintervals, are shown in Figure 4. Approximations to the density using $N=24$ subintervals but different polynomial orders are shown in Figure 5.

6.5. Example 5. Finally, we consider the standard circle map defined by

$$
g_{5}(x)=x+\lambda+\epsilon \sin (2 \pi x) \quad \bmod 1
$$

for which the invariant density is again unknown. The invariant density will be smooth, which we have assumed, if the conjugacy to a rotation is smooth. This is the case if the rotation number satisfies a (complicated) condition [35]. This condition is hard to check but the set of numbers that satisfies it has full Lebesgue measure, and so is likely to be satisfied.

The density for this map was computed by Nicolaisen and Werner [48 for $\lambda=$ 0.3348 and $\epsilon=0.05$. The function $g_{5}$ and the computed density, obtained using a piecewise polynomial approximation consisting of 64 cubic polynomials on $N=256$ subintervals, are shown in Figure6. This density function is in good agreement with that found by Nicolaisen and Werner [4]. 

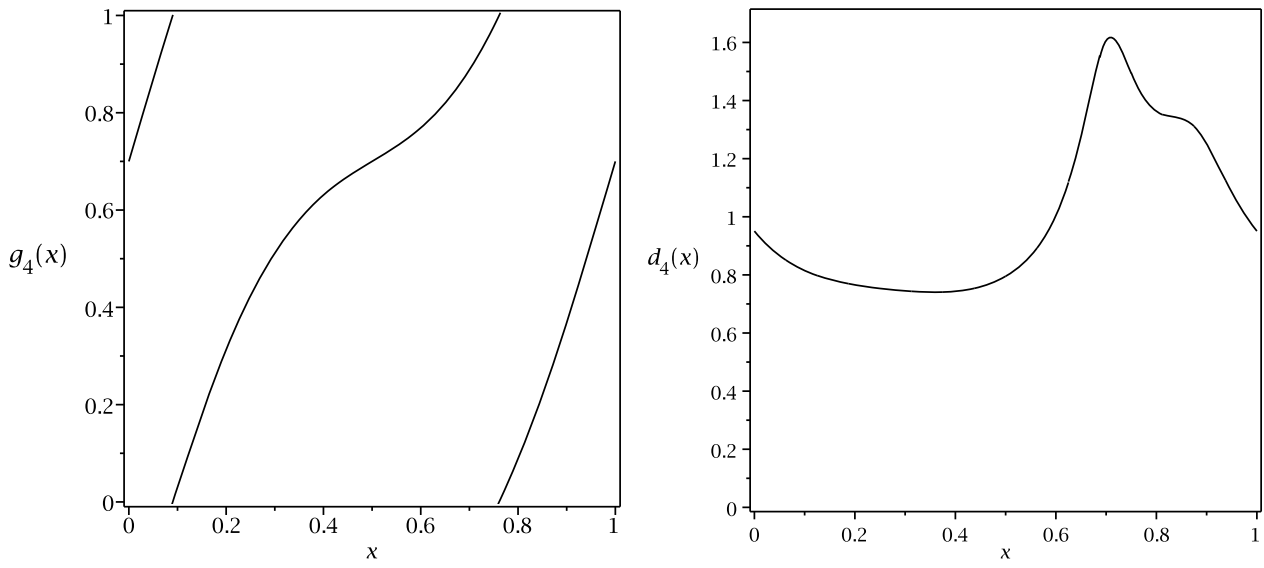

Figure 4. The map $g_{4}$ defined by (6.3) and its density function $d_{4}$ computed using 16 cubic polynomials with $N=64$ subintervals.
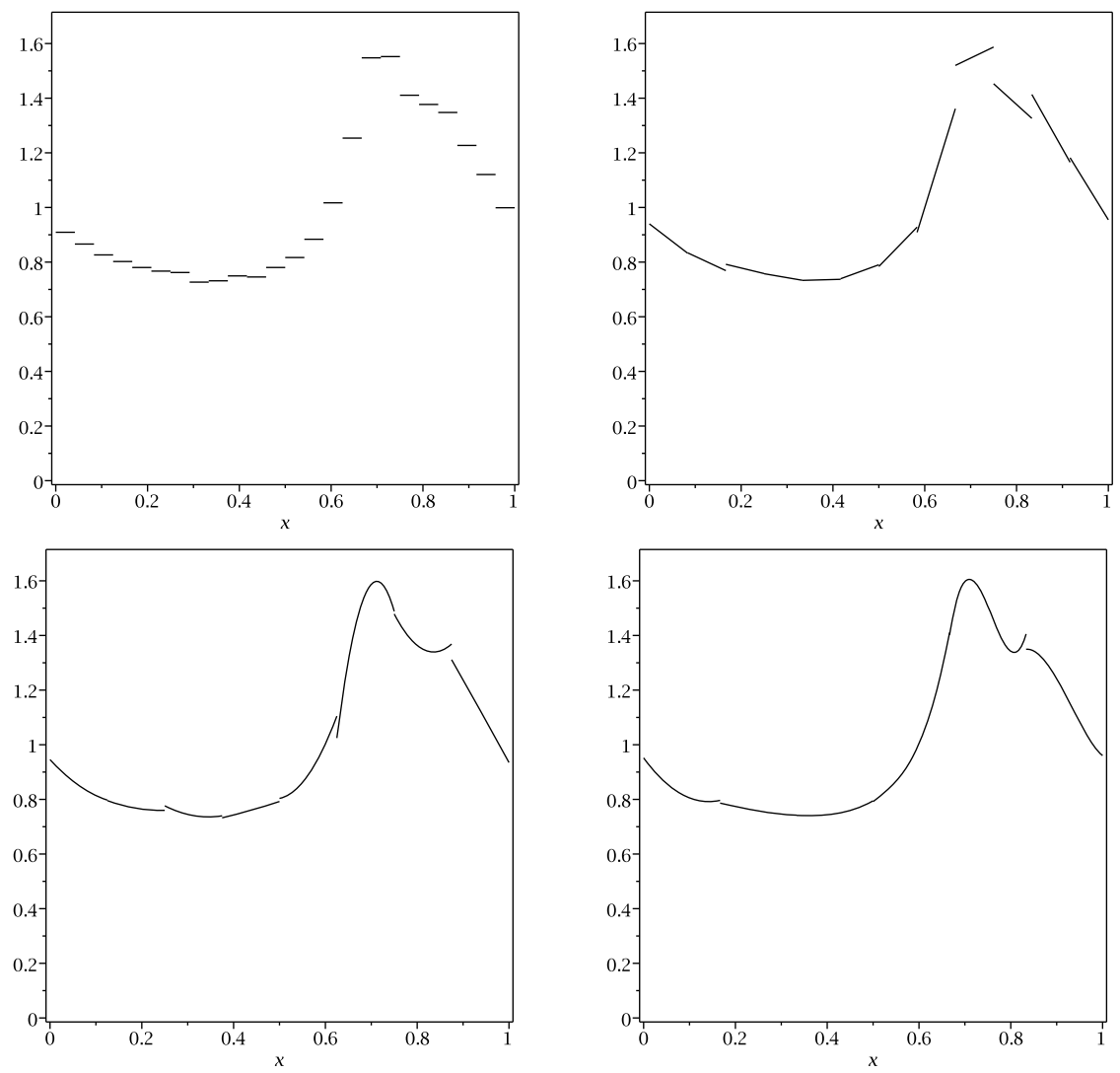

FiguRE 5. Approximation to the invariant density for the map $g_{4}$ with $N=24$ subintervals using (a) 24 constant polynomials $(n=$ $0)$, (b) 12 linear polynomials $(n=1)$, (c) 8 quadratic polynomials $(n=2)$, (d) 6 cubic polynomials $(n=3)$. 

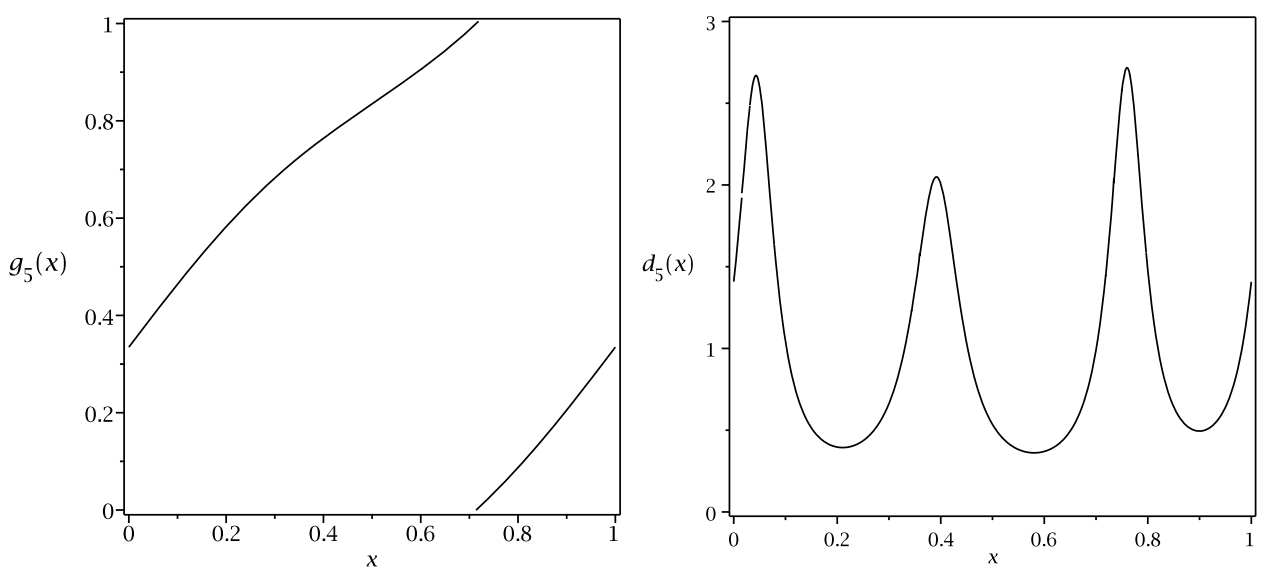

Figure 6. The map $g_{5}$ defined by (6.4) and its density function $d_{5}$ computed using 64 cubic polynomials with $N=256$ subintervals.

The circle map (6.4) is invertible for $|\epsilon|<1 / 2 \pi$, which is the case for our example, and so the motion cannot be chaotic and must therefore be quasiperiodic. The map is not stretching since it is invertible. Similarly, all higher iterates are also invertible and hence are not stretching and so Theorem 5.5 cannot be applied in this case. However, our numerical method still seems to work well and gives a piecewise polynomial approximation that converges to the density for the quasiperiodic orbit.

\section{Computing the Lyapunov exponent using integration}

Having obtained a good approximation to the invariant density, we now want to compute an approximation to the Lyapunov exponent for the map $g$ which is given by

$$
\sigma=\int_{0}^{1} \log \left|g^{\prime}(x)\right| d(x) d x .
$$

We recall from Section 5 that $d_{N}^{n}$ is the discontinuous piecewise polynomial approximation to the invariant density which is a nonzero fixed point of the discretised Frobenius-Perron operator $P_{N}^{n}$ given by (5.4), and that $p_{N}^{n}=L_{N}^{n} d$ is the projection of the true density $d$ onto the piecewise polynomial approximation space. Thus, we can compute $d_{N}^{n}$ as the solution of the discretised Frobenius-Perron equation, but $p_{N}^{n}$ is generally unknown since the true invariant density $d$ is unknown. Then the approximation to the Lyapunov exponent that we can actually compute (ignoring numerical integration errors) is given by

$$
\sigma_{N}^{n}=\int_{0}^{1} \log \left|g^{\prime}(x)\right| d_{N}^{n}(x) d x
$$


A simple error analysis gives that

$$
\begin{aligned}
\left|\sigma-\sigma_{N}^{n}\right| & =\left|\int_{0}^{1} \log \right| g^{\prime}(x)\left|\left(d(x)-d_{N}^{n}(x)\right) d x\right| \\
& \leq \int_{0}^{1}|\log | g^{\prime}(x)\left|\left(d(x)-d_{N}^{n}(x)\right)\right| d x \\
& \leq\left\|\log \left|g^{\prime}\right|\right\|_{\infty}|| d-d_{N}^{n} \|_{1} \\
& =O\left(h^{n+1}\right),
\end{aligned}
$$

using Hölder's inequality and (5.8), assuming that $\log \left|g^{\prime}\right|$ is bounded. Thus, it would appear that the error in the Lyapunov exponent is determined by the error in the invariant density. However, we now show that better results than this can often be obtained.

Theorem 7.1. Assume that $g$ is piecewise $C^{3}[0,1]$ and stretching with $\beta<1$ (cf. (5.7)) and $\gamma_{2}(n) \beta<1$ (cf. (5.6)), and that $\left|g^{\prime}\right| \in C^{0}[0,1]$. If the Frobenius-Perron operator $P$ has a unique invariant density $d \in C^{n+2}[0,1]$ (n odd) or $d \in C^{n+1}[0,1]$ ( $n$ even) then

$$
\left|\sigma-\sigma_{N}^{n}\right|=O\left(h^{n+2}\right)
$$

as $N=1 / h \rightarrow \infty$.

Proof. We consider two different approaches to proving this result.

For the first proof, we note from Corollary [5.6 that

$$
\left\|d-d_{N}^{n}\right\|_{1}=O\left(h^{n+1}\right)
$$

and so we write

$$
d(x)=d_{N}^{n}(x)+h^{n+1} \delta(x)
$$

where $\delta \in L^{1}(0,1)$. Integrating by parts then gives

$$
\begin{aligned}
\left|\sigma-\sigma_{N}^{n}\right|= & \left|\int_{0}^{1} \log \right| g^{\prime}(x)\left|\left(d(x)-d_{N}^{n}(x)\right) d x\right| \\
= & {\left[\log \left|g^{\prime}(x)\right| \int_{0}^{x} d\left(x^{\prime}\right)-d_{N}^{n}\left(x^{\prime}\right) d x^{\prime}\right]_{0}^{1} } \\
& -h^{n+1} \int_{0}^{1} \frac{d}{d x} \log \left|g^{\prime}(x)\right| \int_{0}^{x} \delta\left(x^{\prime}\right) d x^{\prime} d x .
\end{aligned}
$$

Clearly, the boundary term evaluated at $x=0$ is zero and since

$$
\int_{0}^{1} d(x) d x=\int_{0}^{1} d_{N}^{n}(x) d x=1,
$$

by (5.5), then the boundary term evaluated at $x=1$ is also zero. Using the same methods as in the proof of Theorem 4.3, this integration by parts results in the error increasing by one power of $h$ and so the error is $O\left(h^{n+2}\right)$ as claimed.

For the second proof, we recall that $p_{N}^{n}=L_{N}^{n} d$ is the projection of the true invariant density $d$ onto the approximation space $\Delta_{N}^{n}$, where the projection $L_{N}^{n}$ is 
defined by (5.1). Then

$$
\begin{aligned}
\left|\sigma-\sigma_{N}^{n}\right|= & \left|\int_{0}^{1} \log \right| g^{\prime}(x) \mid\left[\left(d(x)-p_{N}^{n}(x)\right)+\left(p_{N}^{n}(x)-d_{N}^{n}(x)\right)\right] d x \\
= & \left|\int_{0}^{1} \log \right| g^{\prime}(x) \mid\left(d(x)-p_{N}^{n}(x)\right) d x \\
& +\int_{0}^{1} \log \left|g^{\prime}(x)\right|\left(p_{N}^{n}(x)-d_{N}^{n}(x)\right) d x \mid \\
\leq & \left|\int_{0}^{1} \log \right| g^{\prime}(x)\left|\left(d(x)-p_{N}^{n}(x)\right) d x\right| \\
& +\left|\int_{0}^{1} \log \right| g^{\prime}(x)\left|\left(p_{N}^{n}(x)-d_{N}^{n}(x)\right) d x\right| .
\end{aligned}
$$

The first integral is of the type that we considered in Section 4.2. The stated conditions on $g$ ensure that $\log \left|g^{\prime}(x)\right|$ satisfies the conditions of Theorem 4.4 and so this theorem gives

$$
\int_{0}^{1} \log \left|g^{\prime}(x)\right|\left(d(x)-p_{N}^{n}(x)\right) d x= \begin{cases}O\left(h^{n+3}\right), & n \text { odd } \\ O\left(h^{n+2}\right), & n \text { even. }\end{cases}
$$

For the second integral, we first note that

$$
p_{N}^{n}-d_{N}^{n}=L_{N}^{n}\left(d-d_{N}^{n}\right)=h^{n+1} \delta_{N}^{n},
$$

using (7.1), where

$$
\delta_{N}^{n}=L_{N}^{n} \delta
$$

Integrating the second integral by parts then gives

$$
\begin{aligned}
\int_{0}^{1} \log \left|g^{\prime}(x)\right|\left(p_{N}^{n}(x)-d_{N}^{n}(x)\right) d x= & {\left[\log \left|g^{\prime}(x)\right| \int_{0}^{x} p_{N}^{n}\left(x^{\prime}\right)-d_{N}^{n}\left(x^{\prime}\right) d x^{\prime}\right]_{0}^{1} } \\
& -h^{n+1} \int_{0}^{1} \frac{d}{d x} \log \left|g^{\prime}(x)\right| \int_{0}^{x} \delta_{N}^{n}\left(x^{\prime}\right) d x^{\prime} d x .
\end{aligned}
$$

Again, the boundary term at $x=0$ is zero and since

$$
\int_{0}^{1} p_{N}^{n}(x) d x=\int_{0}^{1} d_{N}^{n}(x) d x=1,
$$

by (5.5), then the boundary term evaluated at $x=1$ is also zero. Using the same approach now as for the first proof above, the error has increased by one power of $h$ and so this error is $O\left(h^{n+2}\right)$.

Combining these two integrals, asymptotically the largest error is from the second integral and so the error in the Lyapunov exponent is $O\left(h^{n+2}\right)$ as claimed.

7.1. Examples. We consider again the map $g_{1}$ defined by (6.1) that we considered previously in Section 6. We note that for this example, $\left|g_{1}^{\prime}(x)\right|$ is continuous at the point $x=\sqrt{2}-1$, which is one of the conditions of Theorem [7.1.

We used the method described in Section 3 to obtain the piecewise polynomial approximation $d_{N}^{n}(x)$ to the density $d(x)$ and we then evaluated

$$
\sigma_{N}^{n}=\int_{0}^{1} \log \left|g_{1}^{\prime}(x)\right| d_{N}^{n}(x) d x .
$$




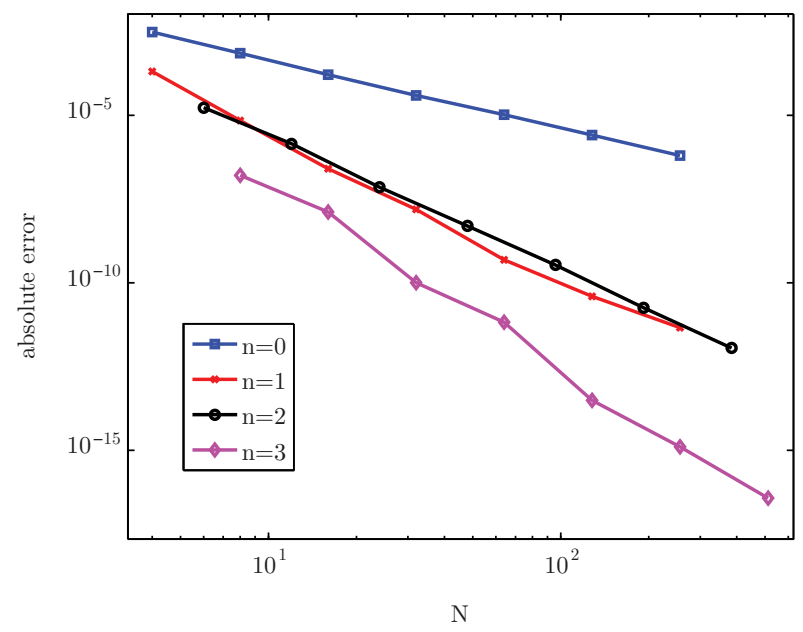

Figure 7. Absolute errors in the computed values of the Lyapunov exponent for the map $g_{1}(x)$.

A fixed point of the discretised Frobenius-Perron operator (5.4) was found numerically using MAPLE which gives the discontinuous piecewise polynomial approximation to the invariant density $d_{N}^{n}$. The approximate Lyapunov exponent $\sigma_{N}^{n}$ given by (7.2) was then computed. The integration was performed accurately by a MAPLE routine rather than using Gaussian quadrature.

Since we know the true density function for this map, we can also accurately compute the true value of the Lyapunov exponent $\sigma$, which is found numerically to have the value $\log 2$. The results of these computations are shown in Figure 7

The slope of the final segment of the lines for $n=0$ and $n=2$ are given by -2.031 and -4.001 , respectively, and so, for even values of $n$, the results agree well with the prediction of Theorem 7.1 that the rate of convergence is $O\left(h^{n+2}\right)$. However, for $n=1$, the rate of convergence initially is very similar to that for $n=2$, which is $O\left(h^{4}\right)$, or equivalently $O\left(h^{n+3}\right)$, and this is higher than that predicted by Theorem 7.1. However, we note that the slope of the final segment of the line for $n=1$ is -3.105 , which is much closer to the predicted rate of convergence of $O\left(h^{3}\right)$. Similarly, for $n=3$, the rate of convergence estimate initially oscillates, but seems to be higher than the prediction of $O\left(h^{5}\right)$. However, considering again the slope of the final segment of the line, we obtain a value of -5.091 , which is close to the predicted rate of $O\left(h^{5}\right)$.

These results can be understood from the second proof of Theorem [7.1. In that proof, the error was broken up into two terms. For $n$ even, the two separate terms were both $O\left(h^{n+2}\right)$. However, for $n$ odd, the terms were of different order so that approximately

$$
\left|\sigma-\sigma_{N}^{n}\right| \approx c_{1} h^{n+3}+c_{2} h^{n+2} .
$$

In this case, the asymptotic rate of convergence as $h \rightarrow 0$ is clearly $O\left(h^{n+2}\right)$. However, the rate of convergence that is observed for finite values of $h$ also depends on the magnitude of the two constants $c_{1}$ and $c_{2}$. If $c_{1} \gg c_{2}$, then for relatively "large" values of $h$, the dominant term in the error will be the first one and so the rate of convergence will appear to be $O\left(h^{n+3}\right)$. However, as $h$ decreases, eventually 
TABLE 3. Approximations to the Lyapunov exponent for the double standard map $g_{4}(x)$ with $N=240$ subintervals.

\begin{tabular}{|c|c|}
\hline$n$ & $\sigma_{240}^{n}$ \\
\hline 0 & 0.5799118772 \\
1 & 0.5799254223 \\
2 & 0.5799191559 \\
3 & 0.5799192357 \\
\hline
\end{tabular}

the higher power of $h$ will ensure that the first term becomes smaller than the second, and then the true rate of convergence of $O\left(h^{n+2}\right)$ will be observed. Clearly, this is what is happening in our example.

We note that even though the $n=1$ and $n=2$ cases have different asymptotic rates of convergence, the actual magnitude of the errors shown in Figure 7 are similar in these two cases. We have noted that for odd $n$, the first integral which is $O\left(h^{n+3}\right)$ seems to dominate the errors for moderate values of $h$, and this implies that the error initially decreases at a similar rate to that for the next even value of $n$. Thus, even though theoretically the results for $n=2$ should be better than those for $n=1$, and would be better for sufficiently small $h$, for moderate values of $h$, the results for these two values are comparable.

Similar results are obtained for the map $g_{2}(x)$ given by (6.2).

For the double standard map $g_{4}(x)$, the density is unknown and so the Lyapunov exponent $\sigma$ is also unknown. Approximations to the Lyapunov exponent using 240 subintervals and different orders of polynomial approximation are shown in Table 3. From these results, comparing the approximate values with the most accurate approximation of $\sigma_{240}^{3}$, we find that $\left|\sigma_{240}^{0}-\sigma_{240}^{3}\right|=7.36 \times 10^{-6}, \mid \sigma_{240}^{1}-$ $\sigma_{240}^{3}\left|=6.19 \times 10^{-6},\right| \sigma_{240}^{2}-\sigma_{240}^{3} \mid=7.99 \times 10^{-8}$ and so again, a significant increase in accuracy of the Lyapunov exponent, is obtained by taking higher values of $n$ for a fixed number of subintervals $N$.

TABLE 4. Approximations to the Lyapunov exponent for the circle map $g_{5}(x)$ with $N=240$ subintervals.

\begin{tabular}{|c|r|}
\hline$n$ & \multicolumn{1}{|c|}{$\sigma_{240}^{n}$} \\
\hline 0 & $-2.7954 \times 10^{-4}$ \\
1 & $3.5636 \times 10^{-7}$ \\
2 & $8.5792 \times 10^{-7}$ \\
3 & $-1.7436 \times 10^{-7}$ \\
\hline
\end{tabular}

Finally, for the circle map $g_{5}(x)$, we note that the Lyapunov exponent is zero since the orbit is quasiperiodic. The rate of convergence for the Lyapunov exponent as proved in Theorem 7.1 depended on the rate of convergence of the density, and in this case, we do not know this rate since Theorem 5.5 does not hold. However, it is found numerically that the computed Lyapunov exponent does converge to zero as either $n \rightarrow \infty$ or $N \rightarrow \infty$. Some results for fixed $N$ but increasing $n$ are shown in Table 4 . 
TABLE 5. The average rotation number for the map $g_{5}(x)$ with $N=240$ subintervals.

\begin{tabular}{|c|c|}
\hline$n$ & Rotation number \\
\hline 0 & 0.3327922036 \\
1 & 0.3327945798 \\
2 & 0.3327944670 \\
3 & 0.3327944188 \\
\hline
\end{tabular}

For this example, of more interest than the Lyapunov exponent, is the rotation number, which is defined by [48]

$$
\rho\left(g_{5}\right)=\int_{x}^{g_{5}(x)} d(x) d x
$$

for all $x \in[0,1)$. When using an approximation to the density, the rotation number does vary slightly for different $x$. Thus, we define

$$
\rho_{N}^{n}\left(g_{5}, x\right)=\int_{x}^{g_{5}(x)} d_{N}^{n}(x) d x
$$

and compute the average of the quantities $\rho_{N}^{n}\left(g_{5}, i h\right), i=0,1, \ldots, N-1$. Some numerical results for these average quantities are shown in Table 5.

\section{Future Directions}

8.1. Stochastic perturbations. By construction, our approach requires the invariant density to be smooth. For deterministic maps, this is not the generic situation. However, in applications one is often faced with a system which is additionally perturbed by (small) random influences. In these cases, instead of the deterministic system $g$, one considers the dynamics

$$
x^{\prime}=g(x)+\xi,
$$

where $\xi$ is chosen from a given probability distribution $\mu$. Suppose that $\mu$ is absolutely continuous with density $h$. Then the associated Frobenius-Perron operator on $L^{1}$ is given by

$$
\operatorname{Pf}(x)=\int h(g(y)-x) f(y) d y, \quad f \in L^{1} .
$$

For common distributions (like a normal distribution or the uniform distribution supported on a small ball around 0) the associated Markov chain possesses finitely many invariant measures which, according to (8.1), inherit the smoothness of the distribution [13].

In this setting, the approach proposed in this paper yields a highly accurate method for the approximation of the invariant distribution. For very small perturbations and in the case of a nonsmooth invariant density, however, one will still need many modes for this. It would be interesting to investigate how the approximation error behaves in dependence on the magnitude of the perturbation. 
8.2. Higher dimensions. The methods described above for one-dimensional maps can easily be generalised to higher dimensions. We briefly consider only the case of a bilinear approximation to the density given the total measure on four neighbouring squares in two dimensions. We assume that a change of variables has been performed so that the region of interest is $[-1,1]^{2}$. We take $t$ and $\tau$ as the two independent variables and we want to approximate the density function $D(t, \tau)$ over this region.

We first define $t_{0}=\tau_{0}=-1, t_{1}=\tau_{1}=0$ and $t_{2}=\tau_{2}=1$ and assume that we know the four values:

$$
M_{i j}=\int_{\tau_{i-1}}^{\tau_{i}} \int_{t_{j-1}}^{t_{j}} D(t, \tau) d t d \tau, \quad i, j=1,2 .
$$

We then want to construct a bilinear approximation to the density which preserves the total measure on each of the four subregions. We write the polynomial approximation as

$$
p_{1}(t, \tau)=\sum_{k, l=1}^{2} M_{k l} \ell_{1, k, l}(t, \tau) .
$$

The basis functions can be determined from the conditions

$$
\int_{\tau_{i-1}}^{\tau_{i}} \int_{t_{j-1}}^{t_{j}} \ell_{1, k, l}(t, \tau) d t d \tau=\left\{\begin{array}{ll}
0, & i \neq k \text { or } j \neq l, \\
1, & i=k \text { and } j=l,
\end{array} \quad i, j=1,2 .\right.
$$

These conditions give rise to the following basis functions:

$$
\begin{aligned}
& \ell_{1,1,1}(t, \tau)=\frac{1}{4}-\frac{1}{2} t-\frac{1}{2} \tau+t \tau, \\
& \ell_{1,1,2}(t, \tau)=\frac{1}{4}+\frac{1}{2} t-\frac{1}{2} \tau-t \tau, \\
& \ell_{1,2,1}(t, \tau)=\frac{1}{4}-\frac{1}{2} t+\frac{1}{2} \tau-t \tau, \\
& \ell_{1,2,2}(t, \tau)=\frac{1}{4}+\frac{1}{2} t+\frac{1}{2} \tau+t \tau .
\end{aligned}
$$

This polynomial basis can then be used to approximate the invariant density, again giving a discontinuous approximation over the whole region. We anticipate that a similar error analysis and convergence analysis as in the one-dimensional case can also be performed.

\section{REFERENCES}

[1] Vítor Araújo, Stefano Luzzatto, and Marcelo Viana, Invariant measures for interval maps with critical points and singularities, Adv. Math. 221 (2009), no. 5, 1428-1444, DOI 10.1016/j.aim.2009.03.004. MR.2522425 (2010e:37050)

[2] Philip J. Aston and Michael Dellnitz, The computation of Lyapunov exponents via spatial integration with application to blowout bifurcations, Comput. Methods Appl. Mech. Engrg. 170 (1999), no. 3-4, 223-237, DOI 10.1016/S0045-7825(98)00196-0. Computational methods and bifurcation theory with applications. MR.1678618(2000b:65143)

[3] E. Borel, Les probabilités dénombrables et leurs applications arithmétiques. Rendiconti del Circolo Matematico di Palermo, 27 (1909), 247-271. DOI 10.1002/BF0301965.

[4] Christopher Bose and Rua Murray, The exact rate of approximation in Ulam's method, Discrete Contin. Dynam. Systems 7 (2001), no. 1, 219-235. MR1806382 (2001m:28028) 
[5] Henk Bruin, Stefano Luzzatto, and Sebastian Van Strien, Decay of correlations in onedimensional dynamics, Ann. Sci. École Norm. Sup. (4) 36 (2003), no. 4, 621-646, DOI 10.1016/S0012-9593(03)00025-9 (English, with English and French summaries). MR2013929 (2005a:37061)

[6] H. Bruin, J. Rivera-Letelier, W. Shen, and S. van Strien, Large derivatives, backward contraction and invariant densities for interval maps, Invent. Math. 172 (2008), no. 3, 509-533, DOI 10.1007/s00222-007-0108-4. MR2393079(2009d:37065)

[7] R. L. Burden and J. D. Faires. Numerical Analysis, 9th edition. Brooks Cole, 2010.

[8] C. Chiu, Q. Du, and T. Y. Li, Error estimates of the Markov finite approximation of the Frobenius-Perron operator, Nonlinear Anal. 19 (1992), no. 4, 291-308, DOI 10.1016/0362546X(92)90175-E. MR.1178404 (93f:28014)

[9] Geon Ho Choe, Computational ergodic theory, Algorithms and Computation in Mathematics, vol. 13, Springer-Verlag, Berlin, 2005. MR2130385 (2005m:37206)

[10] Michael Dellnitz, Gary Froyland, and Stefan Sertl, On the isolated spectrum of the Perron-Frobenius operator, Nonlinearity 13 (2000), no. 4, 1171-1188, DOI 10.1088/09517715/13/4/310. MR 1767953(2001e:37033)

[11] Michael Dellnitz, Andreas Hohmann, Oliver Junge, and Martin Rumpf, Exploring invariant sets and invariant measures, Chaos 7 (1997), no. 2, 221-228, DOI 10.1063/1.166223. MR 1477552 (98g:58099)

[12] M. Dellnitz and O. Junge. An adaptive subdivision technique for the approximation of attractors and invariant measures. Comput. Vis. Sci., 1 (1998), no. 2, 63-68.

[13] Michael Dellnitz and Oliver Junge, On the approximation of complicated dynamical behavior, SIAM J. Numer. Anal. 36 (1999), no. 2, 491-515, DOI 10.1137/S0036142996313002. MR.1668207 (2000c:37026)

[14] Michael Dellnitz, Oliver Junge, Wang Sang Koon, Francois Lekien, Martin W. Lo, Jerrold E. Marsden, Kathrin Padberg, Robert Preis, Shane D. Ross, and Bianca Thiere, Transport in dynamical astronomy and multibody problems, Internat. J. Bifur. Chaos Appl. Sci. Engrg. 15 (2005), no. 3, 699-727, DOI 10.1142/S0218127405012545. MR2136742(2006a:70038)

[15] Peter Deuflhard, Jan Hermans, Benedict Leimkuhler, Alan E. Mark, Sebastian Reich, and Robert D. Skeel, eds., Computational molecular dynamics: challenges, methods, ideas, Lecture Notes in Computational Science and Engineering, vol. 4, Springer-Verlag, Berlin, 1999. MR 1722497 (2000g:81003)

[16] Peter Deuflhard and Marcus Weber, Robust Perron cluster analysis in conformation dynamics, Linear Algebra Appl. 398 (2005), 161-184, DOI 10.1016/j.laa.2004.10.026. MR2121349 (2005h:62166)

[17] J. Ding, Q. Du, and T. Y. Li, High order approximation of the Frobenius-Perron operator, Appl. Math. Comput. 53 (1993), no. 2-3, 151-171, DOI 10.1016/0096-3003(93)90099-Z. MR.1200085 (94d:65036)

[18] J. Ding, Q. Du, and T. Y. Li, The spectral analysis of Frobenius-Perron operators, J. Math. Anal. Appl. 184 (1994), no. 2, 285-301, DOI 10.1006/jmaa.1994.1200. MR.1278389 (95c:47007)

[19] Jiu Ding, Congming Jin, and Aihui Zhou, A posteriori error estimates for Markov approximations of Frobenius-Perron operators, Nonlinear Anal. 67 (2007), no. 3, 763-772, DOI 10.1016/j.na.2006.06.028. MR2319207 (2008f:41031)

[20] Jiu Ding and Tien-Yien Li, Markov finite approximation of Frobenius-Perron operator, Nonlinear Anal. 17 (1991), no. 8, 759-772, DOI 10.1016/0362-546X(91)90211-I. MR1131018 (92i:47008)

[21] Jiu Ding and Tien Yien Li, A convergence rate analysis for Markov finite approximations to a class of Frobenius-Perron operators, Nonlinear Anal. 31 (1998), no. 5-6, 765-777, DOI 10.1016/S0362-546X(97)00438-0. MR1487860 (98j:58090)

[22] Jiu Ding, Tien Yien Li, and Aihui Zhou, Finite approximations of Markov operators, J. Comput. Appl. Math. 147 (2002), no. 1, 137-152, DOI 10.1016/S0377-0427(02)00429-6. MR.1931773 (2003g:37155)

[23] J. Ding and N. H. Rhee, Approximations of Frobenius-Perron operators via interpolation, Nonlinear Anal. 57 (2004), no. 5-6, 831-842, DOI 10.1016/j.na.2004.03.020. MR2067736 (2005c:37035) 
[24] Jiu Ding and Aihui Zhou, Finite approximations of Frobenius-Perron operators. A solution of Ulam's conjecture to multi-dimensional transformations, Phys. D 92 (1996), no. 1-2, 61-68, DOI 10.1016/0167-2789(95)00292-8. MR1384679 (97a:58097)

[25] Jiu Ding and Aihui Zhou, A finite element method for the Frobenius-Perron operator equation, Appl. Math. Comput. 102 (1999), no. 2-3, 155-164, DOI 10.1016/S0096-3003(98)100395. MR1688833 (2000a:65070)

[26] Jiu Ding and Aihui Zhou, Structure preserving finite element approximations of Markov operators, Nonlinearity 15 (2002), no. 3, 923-936, DOI 10.1088/0951-7715/15/3/324. MR.1901114 (2003j:37146)

[27] Gary Froyland, Finite approximation of Sinai-Bowen-Ruelle measures for Anosov systems in two dimensions, Random Comput. Dynam. 3 (1995), no. 4, 251-263. MR.1362773 (96k:58133)

[28] Gary Froyland, Estimating physical invariant measures and space averages of dynamical systems indicators, Bull. Austral. Math. Soc. 56 (1997), no. 1, 157-159, DOI 10.1017/S0004972700030823. (Thesis summary). MR1464058

[29] Gary Froyland, Approximating physical invariant measures of mixing dynamical systems in higher dimensions, Nonlinear Anal. 32 (1998), no. 7, 831-860, DOI 10.1016/S0362546X(97)00527-0. MR.1618409 (99d:58105)

[30] Gary Froyland, Ulam's method for random interval maps, Nonlinearity 12 (1999), no. 4, 1029-1052, DOI 10.1088/0951-7715/12/4/318. MR1709869(2000f:37069)

[31] G. Froyland, K. Padberg, M. England, and A. Treguier, Detection of coherent oceanic structures via transfer operators, Phys. Rev. Lett., 98, 224503, 2007.

[32] Gary Froyland, Naratip Santitissadeekorn, and Adam Monahan, Transport in time-dependent dynamical systems: finite-time coherent sets, Chaos 20 (2010), no. 4, 043116, 10, DOI 10.1063/1.3502450. MR2791360 (2011m:37052)

[33] Rabbijah Guder, Michael Dellnitz, and Edwin Kreuzer, An adaptive method for the approximation of the generalized cell mapping, Chaos Solitons Fractals 8 (1997), no. 4, 525-534, DOI 10.1016/S0960-0779(96)00118-X. MR:1450216(97m:34086)

[34] Ernst Hairer, Christian Lubich, and Gerhard Wanner, Geometric numerical integration, Springer Series in Computational Mathematics, vol. 31, Springer-Verlag, Berlin, 2002. Structure-preserving algorithms for ordinary differential equations. MR1904823 (2003f:65203)

[35] Michael-Robert Herman, Sur la conjugaison différentiable des difféomorphismes du cercle à des rotations, Inst. Hautes Études Sci. Publ. Math. 49 (1979), 5-233 (French). MR538680 (81h:58039)

[36] F. Y. Hunt, A Monte Carlo approach to the approximation of invariant measures, Random Comput. Dynam. 2 (1994), no. 1, 111-133. MR1265229 (95a:58074)

[37] Fern Y. Hunt, Approximating the invariant measures of randomly perturbed dissipative maps, J. Math. Anal. Appl. 198 (1996), no. 2, 534-551, DOI 10.1006/jmaa.1996.0097. MR1376279 (97g:58093)

[38] Fern Y. Hunt, Unique ergodicity and the approximation of attractors and their invariant measures using Ulam's method, Nonlinearity 11 (1998), no. 2, 307-317, DOI 10.1088/09517715/11/2/007. MR1610768(99e:58121)

[39] Fern Y. Hunt and Walter M. Miller, On the approximation of invariant measures, J. Statist. Phys. 66 (1992), no. 1-2, 535-548, DOI 10.1007/BF01060079. MR1149495 (93a:58105)

[40] Oliver Junge, An adaptive subdivision technique for the approximation of attractors and invariant measures: proof of convergence, Dyn. Syst. 16 (2001), no. 3, 213-222, DOI 10.1080/14689360110060708. MR.1851062 (2002j:37123)

[41] Oliver Junge and Péter Koltai, Discretization of the Frobenius-Perron operator using a sparse Haar tensor basis: the sparse Ulam method, SIAM J. Numer. Anal. 47 (2009), no. 5, 34643485, DOI 10.1137/080716864. MR2576507 (2011a:37175)

[42] Jonathan L. King, Three problems in search of a measure, Amer. Math. Monthly 101 (1994), no. 7, 609-628, DOI 10.2307/2974690. MR1289271 (95g:51024)

[43] A. Lasota and James A. Yorke, On the existence of invariant measures for piecewise monotonic transformations, Trans. Amer. Math. Soc. 186 (1973), 481-488 (1974). MR0335758 (49 \#538)

[44] A. Lasota, and P. Rusek, Stability of self-induced vibrations in metal cutting. Proc. the Fifth World Congress on the Theory of Machines and Mechanisms, Montreal, 1979.

[45] Tien Yien Li, Finite approximation for the Frobenius-Perron operator. A solution to Ulam's conjecture, J. Approximation Theory 17 (1976), no. 2, 177-186. MR0412689 (54 \#811) 
[46] Michał Misiurewicz and Ana Rodrigues, Double standard maps, Comm. Math. Phys. 273 (2007), no. 1, 37-65, DOI 10.1007/s00220-007-0223-5. MR2308749 (2008b:37067)

[47] R. Murray. Discrete approximation of invariant densities. PhD thesis, University of Cambridge, 1997.

[48] Nils Nicolaisen and Bodo Werner, Discretization of circle maps, Z. Angew. Math. Phys. 49 (1998), no. 6, 869-895, DOI 10.1007/s000330050127. MR.1664719 (99j:58067)

[49] A. Ralston, On differentiating error terms, Amer. Math. Monthly 70 (1963), 187-189. MR.0157162 (28 \#399)

[50] Anthony Ralston and Philip Rabinowitz, A first course in numerical analysis, 2nd ed., McGraw-Hill Book Co., New York, 1978. International Series in Pure and Applied Mathematics. MR0494814 (58 \#13599)

[51] Ch. Schütte, A. Fischer, W. Huisinga, and P. Deuflhard, A direct approach to conformational dynamics based on hybrid Monte Carlo, J. Comput. Phys. 151 (1999), no. 1, 146-168, DOI 10.1006/jcph.1999.6231. Computational molecular biophysics. MR.1701575 (2000d:92004)

[52] J. F. Steffensen, Interpolation, 2nd ed., Chelsea Publishing Co., New York, NY, 1950. MR0036799 (12,164d)

[53] S. M. Ulam, A collection of mathematical problems, Interscience Tracts in Pure and Applied Mathematics, no. 8, Interscience Publishers, New York-London, 1960. MR0120127 $(22 \# 10884)$

[54] J. H. Wilkinson, The algebraic eigenvalue problem, Clarendon Press, Oxford, 1965. MR0184422(32 \#1894)

Department of Mathematics, University of Surrey, Guildford, Surrey GU2 7XH, UNITED Kingdom

E-mail address: P.Aston@surrey.ac.uk

Technische Universität München, Zentrum Mathematik, Boltzmannstr. 3, D-85747 Garching, Germany

E-mail address: oj@tum.de 